\title{
Equilibrium Properties of the Cellular Automata Models for Traffic Flow in a Single Lane
}

\author{
Alejandro Salcido \\ Instituto de Investigaciones Eléctricas, División de Energías Alternas \\ Mexico
}

\section{Introduction}

In the last thirty years, the application of cellular automata as models of physical systems has attracted much attention, particularly for studying and simulating behaviour of fluid systems and traffic flow. In this work we present a theoretical analysis of the equilibrium properties of the cellular automata models for multi-speed traffic flow in a single lane highway. We hope our studies may advance some steps in the line of establishing a quite well formulated physical theory for these models. Our interest in this problem comes from the believe that general theoretical results about the traffic cellular automata may help very much to improve the speed of the associated computer models that scientists and engineers use for traffic flow simulations; but on another hand, it is much close related to the need of having, in a near future, a simple, but efficient tool for estimating the distribution in space and time of the pollutant emission rates coming from vehicular traffic in urban settlements, in such a way we can use the simulation results as the emissions input for the air pollution dispersion models we use to asses air quality in big urban places like Mexico city.

\subsection{Motivation background and antecedents}

The general development of human societies settled down in urban sites has given new dimensions of all kinds to air pollution the world over during the last few decades. For cities that have became (or are becoming) into geopolitical centres of urban regions with high economic activity, air pollution is a fast growing problem because of the increasing urban population causing high densities of motor vehicle traffic, greater electric power generation needs, and expanding commercial and industrial activities. The high volumes of emissions released to the atmosphere from the urban settlements have such a significant magnitude that a healthy air quality cannot be achieved by natural regeneration (or homeostasis) and scavenging processes only.

A major problem causing high levels of air pollution in big urban settlements, which also increases the complexity of analysis and evaluation of air quality, is the fossil- fuelled urban transport system and its interaction with the city, because motor vehicles produce different emissions under different driving conditions of speed, acceleration and idle. Traffic problems are, in fact, the main culprits of air pollution in urban areas, but that is not the end of the story, because their impacts actually extend even further. The intense traffic of motor vehicles, and their recurrent congestion and jamming produce waste of time and money, increase the risk of car crashing, promote the social unrest, and produce high stress levels 
and health deterioration of the inhabitants of the cities. On another hand, urban traffic is a very complex problem. A growing number of the metropolitan areas world-wide are suffering a transportation demand which largely exceeds capacity. But in many cases, unfortunately, a good enough solution or, even desirable is not simply to extend capacity to meet the demand. Nowadays a coherent handling of the large, and distributed, transportation systems has become in a priority issue in urban planning and management.

Pollution from traffic consists of particulate matter, nitrogen oxides $\left(\mathrm{NO}_{\mathrm{x}}\right)$, carbon monoxide (CO), volatile organic compounds (VOCs), sulphur dioxide $\left(\mathrm{SO}_{2}\right)$, and also other compounds in small amounts, like polyaromatic hydrocarbons (PAH). Lead emission from traffic has reduced dramatically after moving to unleaded fuels. Particulates come from the exhaust emissions, especially from diesel engines, but also from dust and dirt from roads and tires. Other fine particulates are formed by chemical reactions in the atmosphere. Formation of ozone $\mathrm{O}_{3}$ in urban areas is mainly caused by traffic pollutants in a photochemical reaction with UV radiation from the sun.

In the Mexico City Metropolitan Area (MCMA), which is composed of 16 delegations of Mexico City and 59 municipalities of the State of Mexico, the registered vehicular fleet is estimated at more than 4.2 million vehicles. Among these, the $62 \%$ are vehicles registered at Mexico City and the remaining 38\% are units which belong to the State of Mexico. In these figures, private cars account for a significant percentage (80\% in 2006) of the units for the transport of people, and they constitute the most polluting category, producing $52 \%$ of the $\mathrm{CO}, 33 \%$ of the $\mathrm{NO}_{\mathrm{x}}$, and $21 \%$ of the $\mathrm{SO}_{2}$ that are released to the MCMA's atmosphere. Diesel vehicles, particularly trucks and buses, are other important emission sources which contribute, respectively, with the $28 \%$ and $16 \%$ of $\mathrm{PM}_{2.5}$ (particles with diameters $<2.5 \mu \mathrm{m}$ ), and altogether, with $21 \%$ of $\mathrm{NO}_{x}$ (SMA-GDF, 2008).

Against this background, it becomes quite relevant the prediction of the air pollution caused by vehicular sources in urban areas. For this purpose, it is quite important to be able to estimate the space-time distribution of the motor vehicles moving inside an urban area, because, as a matter of methodological order, it is a prior step in estimating the space-time distribution of its respective air polluting emissions. Moreover, since changes in the urban morphology and the spatial distribution of the build in a city can affect traffic flow, and thus the space-time distribution of the vehicle emissions, for the purposes of studying the urban air pollution problems, as well as for the city development planning, it is a quite relevant issue the searching and the developing of simple and reliable tools for simulating vehicular traffic, its emissions and their impacts on air quality. At the MCMA, this is important because, in addition of the daily intense traffic, the urban morphology has changed significantly in last decade, specially by the construction of second floors on the main traffic corridors and explosive growth in the number of skyscrapers and other big buildings.

Air quality analysis for mobile sources, in most cases, is a very complex process which is performed by a combination of several different models. However, although sometimes these models are considered independent of each other, such as it occurs when simply we take the output of one of the models as the input of next one in line, in the real world we find dynamical couplings between processes or phenomena, which cannot be ignored completely in their modelling. Then, it is important to take into account the possible dynamical couplings between the models in integrating the enveloping computational package (or final model) of analysis.

There are basically three types of models required to perform the analysis of the impact of traffic on air quality, as it has been illustrated schematically in Fig. 1. The first type is the 
model describing and projecting the vehicle activities of the facilities to be analyzed. In general, professionals of transportation use two modelling scales, transportation planning models (interested in regional analysis) or traffic flow models (interested in local transportation facilities such as individual roadways, intersections, and ramps, etc.). The second type of analysis (emissions rate models) represents the process of estimating emissions by vehicle fleets. When emission rates are combined with vehicle activity data, the result is an estimate of emissions by time and space. Once the vehicle activities are estimated, and combined with the emissions rates, the atmospheric dispersion of the vehicle emissions can be estimated with a pollution dispersion model. This third type of analysis is performed to estimate pollution concentrations. This final modelling step is needed to estimate pollutant concentrations to which humans are exposed. In this analysis, temporal and spatial estimates of pollutants from transportation and other sources, along with estimates of background pollution and meteorology conditions, are combined. When this analysis is completed, comparisons of estimated pollution concentrations with the National Ambient Air Quality Standards are made to determine whether control action is needed.

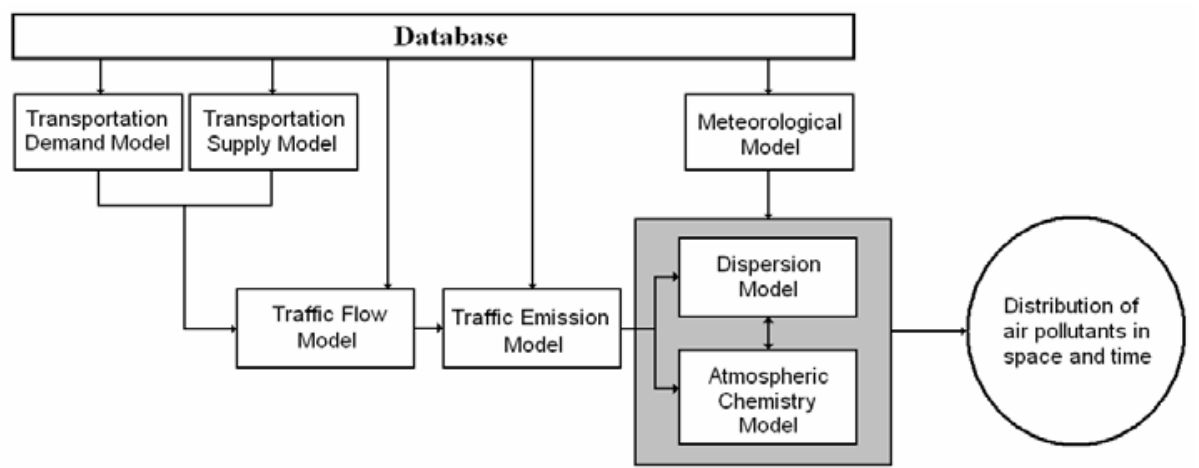

Fig. 1. Simplified combination of models for estimating the impact of traffic flow on air quality. In real world there are feedbacks that complicate the system, such as the influence of transportation supply on land use, or the effect of traffic flows on travel demand in case of congestion.

The knowledge of the wind circulation events constitute an important issue for estimating and understanding how the emissions of air pollutants will be dispersed in an urban settlement and how the air pollution of a city may be exported towards neighbouring sites. Some local pollution dispersion models require only some limited meteorological inputs, but some others may require a detailed knowledge of the wind field, for example. The theoretical basis of meteorological models is in the Navier-Stokes equations, which constitute a system of coupled and non-linear partial differential equations (Batchelor, 1967). For small velocities, these equations can be linearised and solved without much difficulty, analytically if the solid boundaries involved are simple, and numerically otherwise. However, when air flow velocities are large, instabilities may appear and exact analytical methods can no longer be used. Even numerical methods are difficult to use, chiefly because scales of different sizes must be taken into account, which forces grids either to be very small or variable. In practice, a lot of powerful computer simulation tools for diagnostic and prognostic purposes can be found for a variety of applications where the wind field and 
other meteorological variables are required. Notwithstanding, the numerical solutions depend strongly on boundary conditions and initial values; so that special care must be taken to correctly initialise all meteorological variables in the computational domain and to correctly define the time-varying physics at the boundaries (Zannetti, 1990). Two excellent prognostic meteorological models are the PSU/NCAR mesoscale model (MM5) and the Weather Research and Forecasting (WRF) model. These models are complex and heavy numerical simulation instruments adequate only for mesoscale problems (MM5, 2003).

In what concerns to the traffic flow modelling, on the other hand, two different conceptual frameworks are used in general. The oldest one is based on a coarse-grained fluid dynamical description, where traffic is modelled as the flow of a continuum vehicle gas (Kühne, 1998). The other framework is that one of the microscopic traffic models. Here, attention is explicitly focused on individual vehicles, each of which is modelled as a particle that may interact with each other, affecting the others movement. Within this framework, the dynamical evolution of the vehicle gas has been described in terms of several different types of mathematical formulations. For example, a probabilistic description of vehicular traffic has been proposed based on appropriate modifications of the kinetic theory of gases (Prigogine \& Herman, 1971; Helbing, 1996, 1998; Helbing \& Treiber, 1998; Nagatani, 1997a, 1997b), while a deterministic description is provided by the car-following theories based on the Newtonian mechanics (Herman \& Gardels, 1963; Gazis, 1967; Rothery, 1998).

Like the molecular approaches of computational fluid dynamics, microscopic simulation of traffic flow phenomenon has always been regarded as a time consuming complex process involving detailed models that describe the behaviour of individual particles (such as molecules, in the first case, and motor vehicles in the second one). Nevertheless, in the last three decades some conceptually different strategies to simulate the fluid flow and traffic flow phenomena have been developed using the cellular automata techniques introduced by John von Neumann and Stanislaw Ulam in the early 1950s (von Neumann, 1951, 1966).

In the first case, fully discrete models obeying cellular automata rules have been worked out for the microscopic motion of the particles of a gas, such that the coarse-grained behaviour (in the thermodynamic limit) lies in the same universality class as the fluid flow phenomenon. This class of dynamical systems, now known as lattice gas models, consist of a regular lattice, each site of which can have a finite number of states representing the directions of motion of the gas particles, and evolves in discrete time steps obeying a set of homogeneous local rules which define the system dynamics. These rules are defined in such a way that the physical laws of conservation of mass, momentum and energy are fulfilled during the propagation and collisions of the gas particles (Boghosian, 1999). Typically, only the nearest neighbours are involved in the updating of any lattice site.

The first attempt along these lines was undertaken by Leo P. Kadanoff and Jack Swift in 1968 (Kadanoff \& Swift, 1968). The Kadanoff-Swift model exhibits many features of real fluids, such as sound-wave propagation, and long-time tails in velocity autocorrelation functions. As the authors noted, however, it does not faithfully reproduce the correct motion of a viscous fluid (Boghosian, 1999). The next advance in the lattice modelling of fluids came in the mid 1970's, when J. Hardy, O. de Pazzis and Y. Pomeau introduced a new lattice model (the HPP model, named for its authors) with a number of innovations that warrant discussion here (Hardy et al., 1973, 1976). Like the model of Kadanoff and Swift, the HPP model gives rise to anisotropic hydrodynamic equations that are not invariant under a global spatial rotation. At the time, this was not considered a problem, since the real purpose of these models was to study the statistical physics of fluids, and both models could 
do this well. Traditional computational fluid dynamicists, however, were not inclined to take notice of this as a serious numerical method unless and until a way was found to remove the unphysical anisotropy (Boghosian, 1999). Thirteen years passed from the introduction of the HPP model to the solution of the anisotropy problem in 1986 by Uriel Frisch, Brosl Hasslacher and Yves Pomeau (Frish et al., 1986), and simultaneously by Stephen Wolfram (Wolfram, 1986). Frisch, Hasslacher and Pomeau demonstrated that it is possible to simulate the Navier-Stokes fluid flows by using a cellular automata gas model on a hexagonal lattice, with extremely simple translation and collision rules governing the movement of the particles. In the FHP model, named after the authors of the first reference given above, all the particles have unit mass and move with the same speed hopping from site to site in a hexagonal two-dimensional lattice. The dynamics of this system involves a set of collision rules that conserve the number of particles and momentum (kinetic energy is trivially conserved). From a strict theoretical point of view, it is not clear at the present time if the lattice gas collective equations are equivalent to the Navier-Stokes equations, or if they include them as a particular case. However, there has been a growing interest in studying the viscous fluid flow using lattice gas models due to its great facility to handle complex boundary and initial conditions, and also because the computer simulations have shown that lattice gases behave like normal fluids under some restricted conditions (Hasslacher, 1987; Salcido \& Rechtman, 1991, 1993; Rechtman \& Salcido, 1996; Salcido, 1993, 1994). The FHP model, in particular, is now considered as an efficient way to simulate viscous flows at moderate Mach numbers in situations involving complex boundaries. However, it is unable to represent thermal or diffusional effects since all particles have the same speed and are of the same nature (Chen et al., 1989). Maybe the simplest lattice gas with thermal properties is a nine-velocity model defined on a square two-dimensional lattice where particles may be at rest or travelling to their nearest or next nearest neighbours (Chen et al., 1989; Rechtman et al., 1990, 1992; Salcido \& Rechtman, 1991, 1993; Rechtman \& Salcido, 1996).

In the field of air pollution, one of the first attempts to use a cellular automata lattice gas approach for modelling transport and dispersion phenomena of air pollutants can be found in the work by A. Salcido (Salcido, 1993, 1994; Salcido et al., 1993). There, it is shown how the lattice gas rules, in spite of their relative simplicity, are sufficient to simulate, at least qualitatively, some complex processes affecting unsteady dispersion, including momentum exchange with the surrounding atmosphere and deposition. More recent attempts are found in the work by A. Sciarretta and R. Cipollone (Sciarretta \& Cipollone, 2001, 2002; Sciarretta 2006), where a comprehensive stochastic lattice gas model, which provides also reliable quantitative predictions, is presented. Lattice gas approaches to the wind field estimation problem have been developed also (Salcido et al., 2008; Salcido \& Celada, 2010).

Simultaneously with the development of the lattice gas models, a new class of microscopic traffic models emerged also within the conceptual framework of the cellular automata. These new models, known as cellular automata traffic models or traffic cellular automata, are dynamical systems that are discrete in nature, in the sense that the roads are represented by one-dimensional (1D) or two-dimensional lattices, each lattice site being empty or containing exactly one vehicle, and time advances with discrete steps. The first studies in this field were done by Cremer and Ludwig in 1986 (Cremer \& Ludwig, 1986). They proposed a fast simulation model for traffic flow through urban networks. In their model, the progression of cars on a street was simulated by moving 1-bit variables through binary positions of bytes in the storage which were arranged to model the topology of a specified network. Also, in terms of some boolean operations, the model was enabled to perform 
diverse movements of a vehicle, like driving at a constant speed, lane changing, passing, decelerating and accelerating, queueing and turning at intersections. Nevertheless, it was up to the first half of the nineteen nineties, with the proposals of Nagel and Schreckenberg in 1992 (Nagel \& Schreckenberg, 1992) and of Fukui and Ishibashi in 1996 (Fukui \& Ishibashi, 1996a), that cellular automata attracted attention as microscopic traffic models. From then on, traffic scientists have been carrying out many studies about the possibilities of using approaches of cellular automata for building models of traffic that not only are wellformulated from the view of physics and able to reproduce the main behavioural aspects of real vehicular traffic, but also being efficient and practical for computer implementation.

Although traffic cellular automata are quite similar to the cellular automata fluids in several respects, and one can talk about the system like a lattice gas in both cases, in contrast to the fluid models, the particles in a traffic model could be considered, or better yet, would have to be considered as intelligent objects, able to learn from past experience, thereby opening the door to the incorporation of behavioural and psychological aspects (Helbing, 2001; Maerivoet \& De Moor, 2005).

In this chapter, we will not consider the full process of analysis of impact of traffic on urban air quality. Instead, we are interested only in that stage of analysis which is concerned with modelling the traffic flow for the purposes of estimating the distribution of the vehicles (mobile sources) in space and time. Specifically, we will be concerned just with a simple case of this problem, which deals with the simulation of the movement of identical vehicles, but at different speed, in a single lane highway. Within this framework, for example, we would like to be able of finding out the number density of the vehicles which are moving at any given point in the highway, at any given instant, for each speed possible value. So that, by means of an emission rate model, later we would be able to estimate also the distribution in space and time of the vehicular emissions of air pollutants. For these purposes, here we will consider in detail the analysis of the equilibrium properties of the 1D cellular automata traffic models, expecting to provide some general results about this class of traffic models that may contribute not only to improve the speed of the computer simulations, but also in advancing some steps towards a well-established theory of the traffic cellular automata.

In general, it is important to try to address these problems, or any others in this field, starting from the fundamental laws governing the traffic systems behaviour. Using theoretical approaches based on continuum or statistical physics, for more than a half a century physicists have been trying to understand the basic principles governing traffic phenomena and contributing to traffic science by developing models of traffic. The theoretical analysis and computer simulation of these models not only provide deep insight into the properties of the model but also help on improving understanding of complex phenomena observed in real traffic. Moreover, using these models, physicists have been calculating some quantities of interest in practical applications in traffic engineering (Chowdhury et al, 2000).

The rest of this chapter is organized as follows. In the next section, it is provided a general description of the main features and basic aspects of cellular automata and of the cellular automata models for traffic flow in a highway, including presentation and discussion of the main ones with some detail. In section 3 , we presented and discussed the equilibrium theory of the cellular automata models for traffic flow in a single lane, and in the fourth section we provided a detailed comparison of the equilibrium properties of these models against the steady states of the Nagel-Schreckenger and Fukui-Ishibashi traffic cellular automata. Finally, a section devoted to conclusions and suggestions for future work was included. 


\section{Cellular automata and traffic flow models}

At the suggestion of Stanislaw Ulam, cellular automata were introduced by John von Neumann in the early 1950 s as very simple mathematical models to investigate selforganisation and self-reproduction (von Neumann, 1951, 1966). In contrast to the typical mathematical models of self-organisation such as dissipative nonlinear differential equations or iterated mappings, cellular automata provide an alternative approach, involving discrete coordinates and variables as well as discrete time. The main attractive feature of cellular automata is that in spite of their conceptual simplicity, which allows for an easiness of implementation for computer simulation, so as a detailed and complete mathematical analysis in principle, they are able to exhibit a wide variety of amazingly complex behaviour. Thus, numerous physical and other systems containing many discrete elements with local interactions, for example the dynamical Ising model, gas and fluid dynamics, traffic flow, various biological issues, growth of crystals, nonlinear chemical systems, and some many others, can be conveniently modelled as cellular automata (Toffoli, 1984; Doolen et al, 1990; Chopard \& Droz, 1998; Wolfram, 1986b, 1994, 2002; Bagnoli, 2001; Stauffer, 2001, Maerivoet \& De Moor, 2005).

\subsection{Main features of cellular automata}

In order to understand why and how cellular automata can be used as models for various systems in nature, we will begin by describing very briefly the main ingredients that constitute a cellular automaton: the physical environment, the states of the sites, their neighbourhoods, and finally a local transition rule. More complete and detailed descriptions can be found, for example, in the works of F. Bagnoli (Bagnoli, 2001) and B. Chopard and M. Droz (Chopard \& Droz, 1998).

Cellular automata are fully discrete dynamical systems. The physical environment of a cellular automata system is constituted of a finite-dimensional lattice, with each site (cell or box) having a finite number of discrete states. The state of the system is completely specified by the states at each lattice site. It evolves in time in discrete steps, and its dynamics is specified by some fixed and definite rule of evolution, which may be deterministic or nondeterministic (probabilistic), and, in general, may have many simplifying features: it is homogeneous (all sites evolve by the same rule) although inhomogeneous cellular automata can been considered too; it is spatially local (the rules for the evolution of each site depend only on the state of the site itself and the states of sites in its local neighbourhood); it allows for synchronous updating (all cells can be updated simultaneously); it is temporally local (the rule depends only on cell values at the previous time-step, or a few previous ones). Figure 2 illustrates the most common neighbourhoods used with cellular automata defined on one and two-dimensional lattices.

For 2D (and higher dimensional) cellular automata, the number of nearest and next nearest lattice sites contained in the neighbourhood depends on the lattice topology. In Fig. 2, it was illustrated only the case of a square 2D lattice.

The entirely local construction of cellular automata has for a crucial consequence the fact that cellular automata rules define no intrinsic length scale other than the size of a single site and its neighbourhood, and no intrinsic time scale other than the duration of a single time step. In the infinite time limit the configurations are self-similar, and views of the configuration with different magnifications are indistinguishable. 


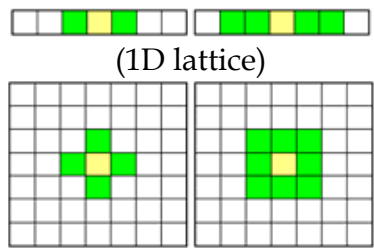

(Square 2D lattice)

Fig. 2. Neighbourhoods commonly used with 1D and 2D cellular automata. The von

Neumann neighbourhood (left) consists of the central site itself (in yellow) plus the nearest neighbours (in green), and the Moore neighbourhood (right) is composed of the central site itself (yellow) plus the nearest and next nearest neighbours (in green).

In practice, the computer simulations using cellular automata models are carried out on a finite rather than an infinite lattice, and therefore it is important to consider how to handle the sites on the edges, as this can affect the values of all the sites in the lattice. It is possible to define neighbourhoods differently for the sites on the boundary, but then new rules for them have to be defined as well. Another possibility is fixing the values of these sites to remain constant, corresponding to Dirichlet boundary condition for partial differential equations. Most often periodic boundary conditions are assumed, where the first and the last sites are identified; for instance, in one dimension the lattice sites are treated as if they lay on a circle of finite radius, and similarly for higher dimensions. This also solves the boundary problems with neighbourhoods.

Despite their conceptual simplicity, cellular automata are capable of diverse and complex behaviour. For most cellular automata models, however, the only general method to determine the qualitative (average) dynamics of the system is to run simulations on a computer for several initial global configurations. Cellular automata classification based on the study of its dynamics has been a major focus for the researchers. Simulations suggest that the patterns generated in the time evolution of cellular automata from disordered initial states can be classified as follows (Wolfram, 1984): Class 1: cellular automata which evolve to a homogeneous state; Class 2: displaying simple separated periodic structures; Class 3: which exhibit chaotic or pseudo-random behaviour; and Class 4: which yield complex patterns of localized structures and are capable of universal computation.

\subsection{Traffic cellular automata}

The application of cellular automata to traffic dynamics goes back to Cremer and coworkers (Cremer and Ludwig, 1986; Schütt, 1991), to Nagel and Schreckenberg (Nagel \& Schreckenberg, 1992), and to Fukui and Ishibashi (Fukui \& Ishibashi, 1996a). Other early cellular automaton studies were carried out by Biham et al. (Biham et al., 1992). These proposals of microscopic traffic models stimulated an enormous amount of research activity, aimed at understanding and controlling traffic instabilities, which are responsible for stop-and-go traffic and congestion, both on freeways (Sasvári \& Kertész, 1997) and in cities (Helbing, 2001). Since then, cellular automata became popular for the microscopic simulation of traffic flow (Vilar \& de Souza, 1994; Chowdhury et al, 2000; Helbing, 2001; Nagatani, 2002; Nagel et al., 2003; Maerivoet \& De Moor, 2005), including multilane highways (Wagner et al., 1997; Nagel et al., 1998; Chowdhury et al., 2000; Nagel et al., 2003; Maerivoet \& De Moor, 2005) and complex urban traffic networks (Fukui \& Ishibashi, 1996b; Fukui et al., 1996; Esser \& Schreckenberg, 1997; Rickert \& Nagel, 1997; Nagel \& Barrett, 1997; 
Simon \& Nagel, 1998; Maerivoet \& De Moor, 2005). Nowadays, there exist an overwhelming number of proposals and publications in this field.

Here, however, we will focus our interests on the cellular automata models for unidirectional single-lane traffic flow with periodic boundary conditions. Some insight to the importance of studying this basic problem can be obtained by considering, for example, traffic flows on unidirectional two-lane motorways: Drivers, in many countries, are by law obliged to drive on the right hand lane, unless when performing overtaking manoeuvres. A frequently observed phenomenon is then that under light traffic conditions, a slower moving vehicle is located on the right lane, and is acting as a moving bottleneck. As a result, all faster vehicles will line up on the left lane (overtaking on the right lane is prohibited by law), thereby causing a population inversion in the lanes. It is under these circumstances that the stability of the car-following behaviour plays an important role (Maerivoet \& De Moor, 2005). Even for multi-lane traffic, its dynamics is essentially that of parallel single lanes when considering densely congested traffic flows. Studying these simplified traffic flow conditions is, in fact, the easiest way to determine whether or not internal effects of a traffic flow model play a role in, for example, the spontaneous breakdown of traffic, as all external effects (i.e., the boundary conditions) are eliminated (Nagel \& Nelson, 2005). Nevertheless, when applying these models to real-life traffic networks, closed-loop traffic is not very representative, as the behaviour near bottlenecks plays a far more important role (Helbing, 2001).

\subsubsection{Common features in cellular automata models for traffic flow in a single-lane}

For the basic problem of traffic flow of identical vehicles in a single-lane, there are three cellular automata models that we consider important for our purposes in this work: the model defined by the Wolfram's rule CA-184, and the original models proposed by Nagel and Schreckenberg (Nagel \& Schreckenberg, 1992) and by Fukui and Ishibashi (Fukui \& Ishibashi, 1996a). These models (hereafter referred as WR184, NS and FI, respectively), so as most of cellular automata models for unidirectional single-lane traffic flow, have the following basic common characteristics: each of them can be considered as a 1D lattice gas of undistinguishable particles with unit mass (model cars) which obey an exclusion principle (no more than one particle may occupy any lattice site at any time), can be at rest or moving with positive integer velocities $v$ up to an upper limit $v_{\max }$ (reverse motion is forbidden and there exists an speed limit), and interact each other according to a specific set of parallel updating-rules (applied synchronously to all particles) that conserve the number of particles and prevent collisions (car crashes) and overtaking, but do not conserve momentum and energy of the particles. The main difference between these models is concerned with the particular procedure that is implemented to change the speed of the lattice gas particles. In the next three subsections we describe the main features of the sets of rules (updating rules) of the models WR184, NS and FI that are consecutively applied to all vehicles in the lattice.

\subsubsection{The Wolfram's CA184 traffic model}

The simplest one-dimensional cellular automata model for highway traffic flow is the model defined by the Wolfram's rule CA-184. This is a deterministic cellular automata model whose dynamics is defined by the following two rules:

R1. Acceleration and braking: $\quad v_{\mathrm{i}}(t+1) \leftarrow \min \left\{h_{\mathrm{i}}(t), 1\right\}$

R2. Vehicle movement: $\quad x_{\mathrm{i}}(t+1) \leftarrow x_{\mathrm{i}}(t)+v_{\mathrm{i}}(t+1)$ 
Rule R1 sets the speed $v_{\mathrm{i}}$ of the i-th vehicle, for the current updated configuration of the system; it states that a vehicle always strives to drive at a speed of one lattice site per timestep, unless its impeded by its direct leader, in which case $h_{\mathrm{i}}(t)$, the number of empty sites in front of the i-th vehicle at time $t$, is equal zero, and the vehicle consequently stops in order to avoid a collision. The rule R2 just allows the vehicles to advance in the lattice.

The Wolfram's rule 184 can be expressed also as follows. The state of each lattice site at any time is expressed by a 1-digit binary number, whose value is 1 if the site is occupied by a particle and 0 otherwise. For any lattice site, $i$, the state at time $t+1$, denoted by $\sigma(i, t+1)$, will be a function of the states $\sigma(i-1, t), \sigma(i, t)$, and $\sigma(i+1, t)$, at time $t$, in the sites which compose the Moore neighbourhood of the site in question, $\mathcal{N}_{\mathrm{i}}=\{i-1, i, i+1\}$. The configuration of the states of the sites in $\mathcal{N}_{\mathrm{i}}$ is expressed as a 3-digit binary number $\xi(i, t)=\sigma(i-1, t) \sigma(i, t) \sigma(i+1, t)$. Then the evolution in time of the state at the lattice site $i$ can be written as

$$
\sigma(i, t+1)=\mathscr{F}(\xi(i, t))
$$

where the function $\mathcal{F}$ is defined by the updating rule given in Table 1.

\begin{tabular}{|c|c|c|c|c|c|c|c|c|}
\hline$\xi(\mathrm{i}, \mathrm{t})$ & 111 & 110 & 101 & 100 & 011 & 010 & 001 & 000 \\
\hline$\sigma(\mathrm{i}, \mathrm{t}+1)$ & 1 & 0 & 1 & 1 & 1 & 0 & 0 & 0 \\
\hline
\end{tabular}

Table 1. Wolfram's rule 184. All eight possible configurations for the local neighbourhood are sorted in the first row, and the results are shown in the second row. The physical meaning is that a particle (a 1) moves to the right if its right neighbouring site is empty.

In Fig. 3, we show the evolution in time of the traffic model WR184. We considered a lattice consisting of 500 sites with periodic boundary conditions, and carried out simulations over a period of 465 timesteps each, for mean densities $n=0.15,0.25,0.35,0.45,0.5,0.55,0.65,0.75$, and 0.85 particles/site. Each case, the initial condition was prepared by distributing the particles randomly in the lattice.

In the figures, the time and space axes are oriented from left to right, and top to bottom, respectively. The simulations show the occurrence of a free-flow regime for low densities (first row); a transition from a free-flow to a congested-flow regime for densities around the critical density $n_{c}=0.50$ particles/site (second row); and a congested-flow regime for high densities (third row). As time advances, the congestion waves can be seen propagating in the opposite direction of traffic. We can see also that the WR184 model constitutes a fully deterministic system that continuously repeats itself. A characteristic of the encountered congestion waves is that they have an eternal life time.

Let $n_{0}(t)$ and $n_{1}(t)$ denote the average numbers of particles per lattice site at time $t$, which are at rest $\left(c_{0}=0\right)$ and moving with the speed one $\left(c_{1}=1\right)$, respectively. Then the mean flow is given by $q=n_{0} c_{0}+n_{1} c_{1}=n_{1}$, and the mean speed is $v=q / n=n_{1} / n$, where $n=n_{0}+n_{1}$ is the mean density of particles in the lattice. In Fig. 4, there are shown the plots of $n_{0}, n_{1}, q$ and $v$ as functions of $n$ for the steady state of the WR184 model. As can be seen from the plot drawn in green, the mean speed remains constant at $v=c_{1}=1$ sites per timestep, until the critical density $n_{c}=0.5$ particles/site is reached, at which point $v$ will start to diminish towards zero where the density $n=1$ particles/site is reached. Similarly, the mean flow $q$ (plot drawn in red) first increases and then decreases linearly with the density, below and respectively above, the critical density. Here, the capacity flow $q_{\text {cap }}=0.5$ particles/timestep is reached. The transition from the free-flowing to the congested regime is characterised by a 
population inversion from the particles in motion (with density $n_{1}$; plot drawn in red) to the particles at rest (with density $n_{0}$; plot drawn in blue). As is evidenced by the isosceles triangular shape of the fundamental diagram ( $q$ as function of $n$ ) of the WR184 traffic model, there are only two possible kinematic wave speeds: $c_{w}= \pm 1$ site/timestep. Both speeds are also clearly visible in the first row, respectively third row, time-space diagrams of Fig. 3.
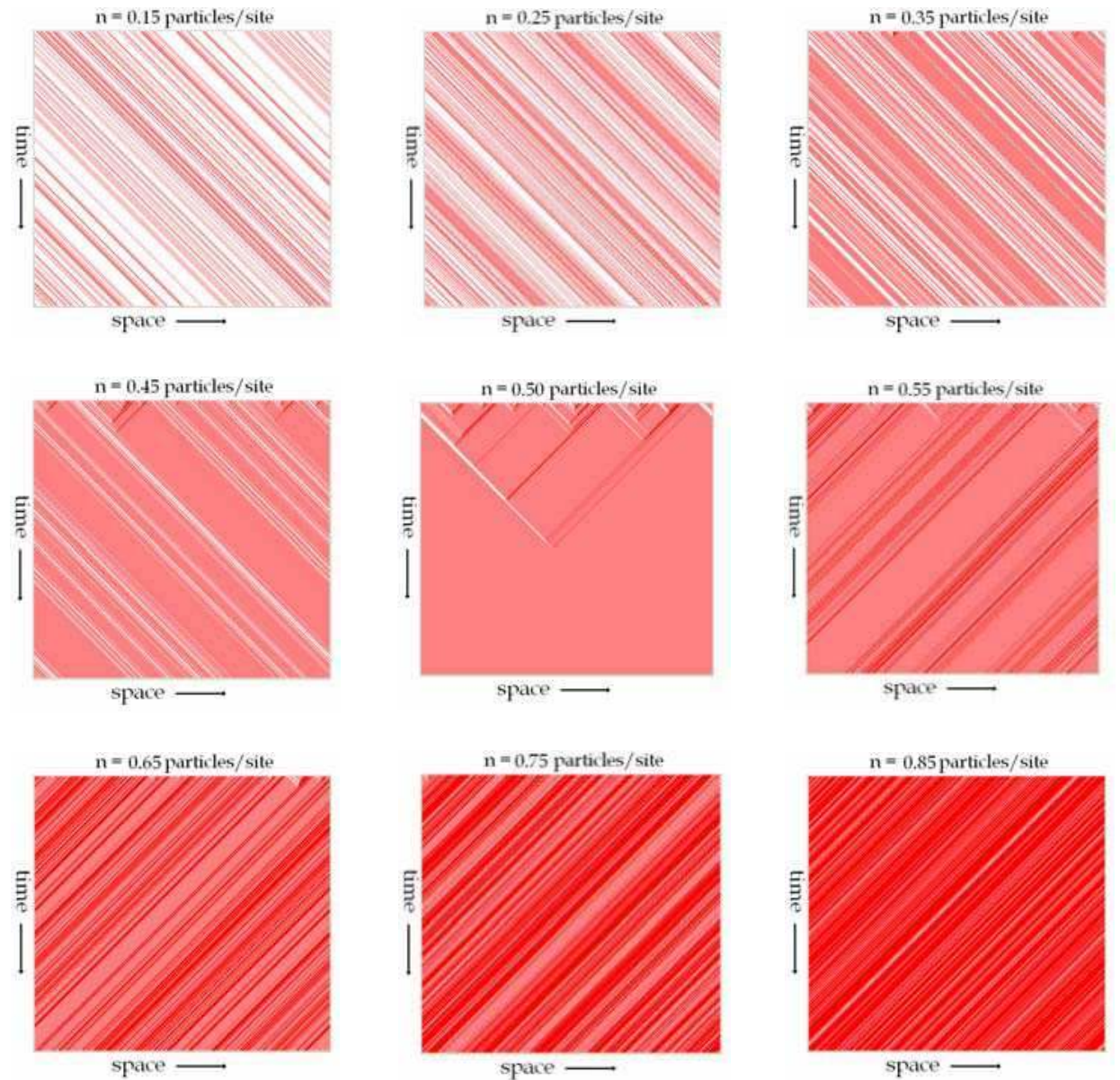

Fig. 3. Typical time-space diagrams of the WR184 traffic model. The shown ring-geometry lattices each contain 500 sites, with a visible period of 465 timesteps (each vehicle is represented as a single coloured dot). First row: vehicles driving a free-flow regime with mean densities $n=0.15,0.25$ and 0.35 particles/site. Second row: a transition from the free-flow regime to the congested one, occurring for densities around $n=0.50$ particles/site. Third row: vehicles driving in a congested regime with $n=0.65,0.75$ and 0.85 particles/site. The congestion waves can be seen as propagating in the opposite direction of traffic. 


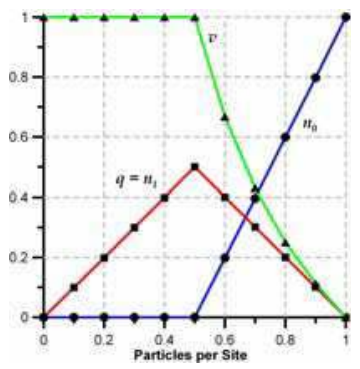

Fig. 4. Typical behaviour diagrams of the WR184 model, based on global measurements on the lattice carried out at the steady state. Green $(\boldsymbol{\Delta})$ : mean speed remains constant at $v=1$ site/timestep, until the critical density $n_{c}=0.5$ is reached, at which point $v$ will start to diminish towards zero. Red ( $\mathbf{m})$ : flow diagram, with its characteristic isosceles triangular shape. The transition between the free-flowing and the congested regimes is observed. Blue $(\bullet)$ : the number of particles at rest remains null $\left(n_{0}=0\right)$ until the critical density is reached, at which point it starts to increase towards one $\left(n_{0}=1\right)$. The transition between the freeflowing and the congested regimes is close related to a population inversion between moving particles and particles at rest (plots identified by symbols ( $\bullet$ ) and $(\bullet)$, respectively).

\subsubsection{The Nagel-Schreckenberg traffic model}

In 1992, Kai Nagel and Michael Schreckenberg proposed a very simple stochastic cellular automata traffic model (Nagel \& Schreckenberg, 1992). In the NS model, space and time are discrete and hence also the velocities. The road, which is supposed unidirectional, is modelled by a $1 \mathrm{D}$ lattice with $L$ sites (cells or boxes) that represent the positions of the vehicles. The number of sites in the lattice may be considered finite or infinite. The distance between adjacent lattice sites is defined as unit in this work, although it is often determined by the front-bumper to front-bumper distance of cars in the densest jam and is usually taken to be $7.5 \mathrm{~m}$. Each site can either be empty or occupied by one, and only one particle (car or vehicle), which can be at rest $(v=0)$ or moving along the lattice (always in the same direction, hereafter assumed from left to right) with a integer speed $v=1,2,3, \ldots, v_{\max }$. The evolution of the system in time (its dynamics) is defined by the following four rules, which must be applied to all particles (i.e. to all the non-empty lattice sites) simultaneously (Nagel \& Schreckenberg, 1992). If at time $t$, there is a particle at site $k(k=1,2,3, \ldots, L)$, then

R1. Acceleration: the particle's speed $v(k, t)$ is substituted by the smallest of $v(k, t)+1$ and $v_{\max }$. That is: $v(k, t) \rightarrow u(k, t)=\min \left\{v(k, t)+1, v_{\max }\right\}$

R2. Braking: if $d(k, t)$, the number of the empty sites ahead the particle at time $t$, is smaller than $u(k, t)$, then $u(k, t) \rightarrow w(k, t)=\min \{d(k, t), u(k, t)\}$

R3. Randomization: with probability $p$, the speed of the particle at time $t+1$ is set equal to the largest of $w(k, t)-1$ and 0 . That is: $v(k, t+1)=\max \{w(k, t)-1,0\}$

R4. Driving: the particle moves hopping from site $k$ to site $k+v(k, t+1)$.

The number of empty sites in front of a car is called headway. For $v_{\max }=5$ a calibration of the model showed that each timestep $t \rightarrow t+1$ corresponds to approximately $1 \mathrm{sec}$ in real time (Nagel \& Schreckenberg, 1992). Hereafter we will consider only a lattice with periodic boundary conditions, so that the number of particles is conserved. The maximum velocity $v_{\max }$ can be interpreted as a speed limit that drivers are obligated to respect, and therefore it will be taken to be identical for all particles. Fig. 5 shows a typical configuration. 


\begin{tabular}{|c|c|c|c|c|c|}
\hline$\infty$ & $\infty$ & $\infty \infty$ & $\infty$ & $a \infty a$ & $a \infty a \infty a$ \\
\hline
\end{tabular}

Fig. 5. A possible configuration during the time evolution of the Nagel and Schreckenberg traffic model. The lattices sites have been drawn with different colors for evidencing the speeds of the vehicles.

The four updating rules of the NS model have simple interpretations within the traffic jargon context (Schadschneider, 1999). The first rule (R1) means that drivers want to drive as fast as allowed. The rule R2 means that drivers have to brake to avoid collision with the vehicle ahead. The rule R3 (randomization) takes into account several effects, e.g. road conditions (e.g. slope, weather) or psychological effects (e.g. velocity fluctuations in free traffic). An important consequence of this rule is the introduction of overreactions at braking which are crucial for the occurrence of spontaneous jam formation (Schadschneider, 1999). Although this rationale is widely agreed upon, much criticism was however expressed due to the rule $\mathrm{R} 3$. In particular, Brilon and $\mathrm{Wu}$ believe that this rule has no theoretical background and is in fact introduced quite heuristically (Brilon \& Wu, 1999). The last rule (R4) implements the displacement of the vehicles. Thus the NS model captures the features of gradual acceleration, deceleration and randomization in realistic traffic flows and, in agreement with the results of the computer simulations, it seems that all four rules, R1-R4, are necessary to reproduce the basic properties of real traffic; therefore this model is considered as a minimal model. An intuitive feeling for the NS model dynamics can be obtained from the nine time-space diagrams presented in Fig. 6.

The diagrams in Fig. 6 were obtained as follows. We considered a lattice consisting of 500 sites with periodic boundary conditions, and carried out simulations over a period of 465 timesteps each. In the figure, we have arranged these diagrams in a $3 \times 3$ matrix, for illustrating several aspects of the evolution of the NS traffic model with $v_{\max }=1$. The matrix rows correspond to mean densities $n=0.25,0.50$ and 0.75 particles per site, and the columns correspond to randomization probabilities $p=0.25,0.50$ and 0.75 . In the figures, the time and space axes are oriented from left to right, and top to bottom, respectively. As can be seen in the diagrams, the randomization rule (R3) gives rise to many unstable artificial phantom mini-jams. The downstream fronts of these jams smear out, forming unstable interfaces (Nagel et al., 2003). This is a direct result of the fact that the intrinsic noise (as embodied by $p$ ) in the NS model is too strong: a jam can always form at any density, meaning that breakdown will occur, even in the free-flow traffic regime. For low enough densities however, these jams can vanish as they are absorbed by vehicles with sufficient space headways or by new jams in the system (Krauß, et al., 1999).

In Fig. 7, for the NS model with $v_{\max }=1$, and in Fig. 8, for $v_{\max }=2$ (top row) and $v_{\max }=3$ (bottom row), there are shown steady-state simulation results for the mean flow $q$ and the partial densities $n_{v}$ (global average numbers of the particles per site which move with the speed $v$ ) as functions of $n$, for randomization probabilities $p=0.0,0.25$ and 0.50 . The simulations were carried out using a 860 -sites lattice with periodic boundary conditions, and proceeding as follows: for density values increasing from 0 to 1 with steps of $\Delta n=0.01$, the system was allowed to evolve for 1000 timesteps, and each simulation run was repeated 20 times. As can be seen in Fig. 7, although the NS model with $v_{\max }=1$ and $p=0$ has exactly the same behaviour as the WR184 model, important and growing deviations from this model become evident as $p$ increases from zero. The top and bottom rows of Fig. 8 show the steady state behaviour of the NS model for $v_{\max }=2$ and $v_{\max }=3$, respectively, for three 
values of the parameter $p$. In both cases, when $p=0$ the system remains under the freeflowing regime (all the particles moving with the maximum speed) until the mean density $n$, growing from zero, reaches the critical densities $n_{c}=1 / 3$ and $n_{c}=1 / 4$, respectively.

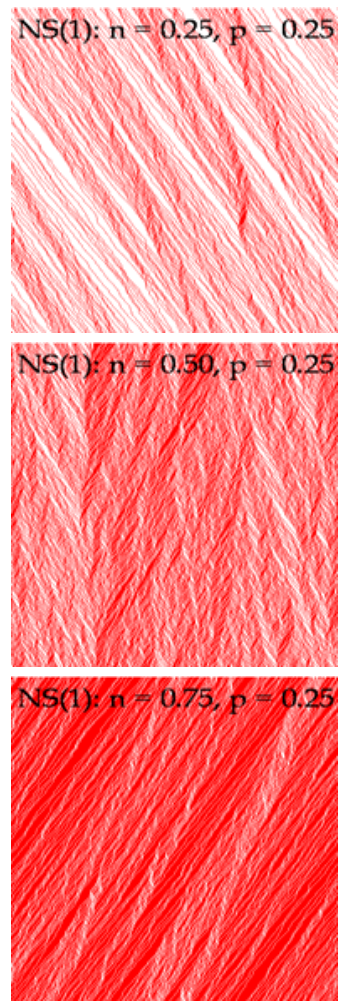

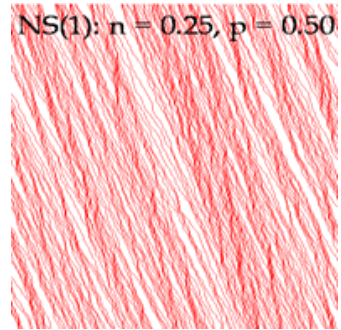
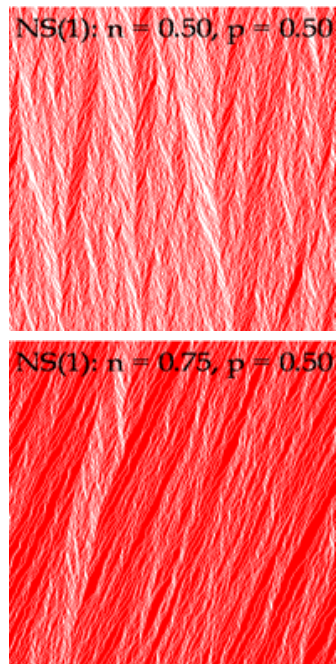
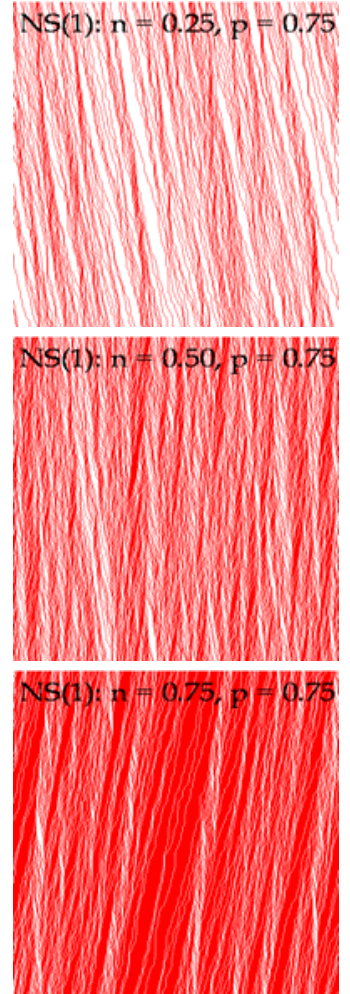

Fig. 6. Time-space diagrams showing the behaviour of the Nagel and Scherckenberg traffic model for several values of density $n$ and randomization parameter $p$. Simulations were carried out on a 500 sites lattice with periodic boundary conditions, for periods of 465 timesteps.
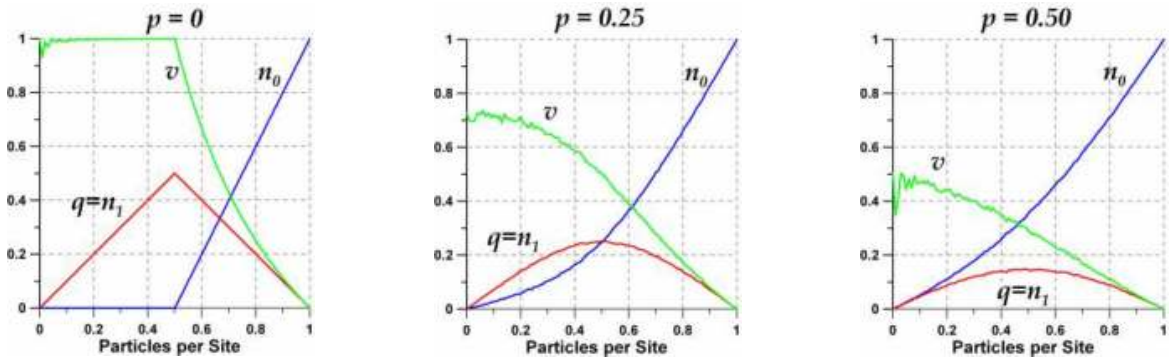

Fig. 7. Steady-state behaviour of the NS model with $v_{\max }=1$. The diagrams show the effect of the randomization $p$ on the mean speed $v$ and the partial densities $n_{v}$ as functions of density $n$. This model with $p=0$ is exactly the same as the WR184 model. 


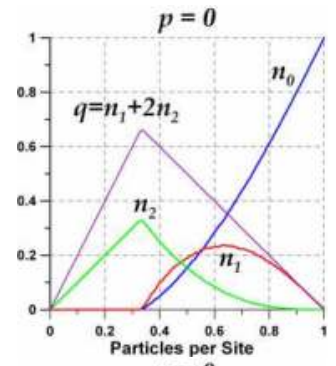

$p=0$

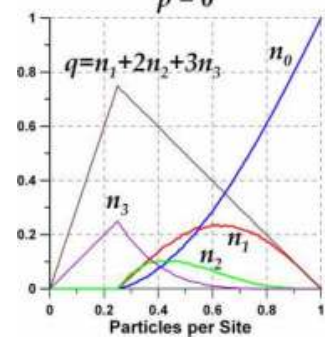

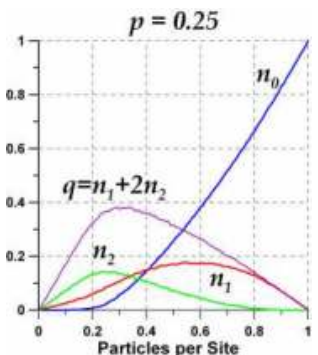

$p=0.25$

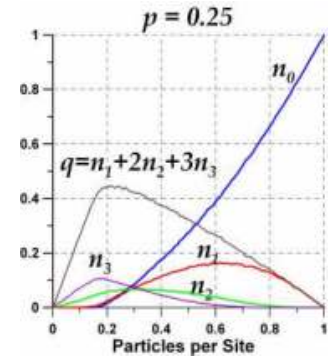

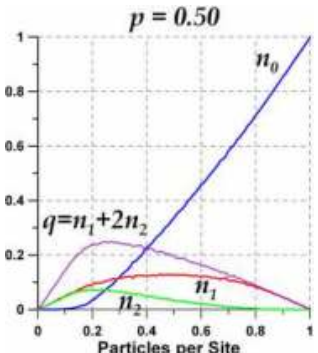

$p=0.50$

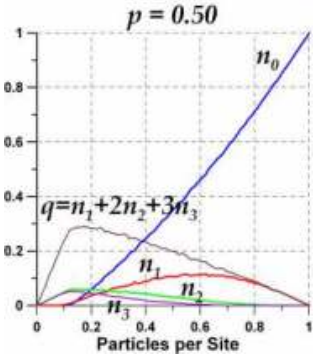

Fig. 8. Steady states of the NS model with $v_{\max }=2$ (top row) and $v_{\max }=3$ (bottom row) for randomization probabilities $p=0.00,0.25$ and 0.50 . For $p=0$ the system remains under a free-flowing regime until density $n$ reaches the values $n_{c}=1 / 3$ and $n_{c}=1 / 4$, respectively.

\subsubsection{The Fukui-Ishibashi traffic model}

In 1996, M. Fukui and Y. Ishibashi (Fukui \& Ishibashi, 1993, 1996a; Wang et al., 1997) proposed another cellular automata model for traffic flow in a single lane (hereafter referred as FI), where the cars can move by at most $v_{\max }$ lattice sites in one timestep if they are not blocked by cars in front. In detail, if the number of empty sites $h$ in front of a car is larger than $v_{\max }$ at time $t$, then it can move forward $v_{\max }$ (or $v_{\max }-1$ ) sites in the next time-step with probability 1 - $f$ (or $f$ ). Here, the probability $f$ represents the degree of stochastic delay. From the point of view of this model, no driver would like to slow down when far away from the vehicle ahead. In the high density case, the stochastic delay in this model represents the assurance of the avoidance of crashes. The model with $f=0$ is referred to as the deterministic FI model with maximum speed $v_{\max }$, while the case with $f=1$ is the deterministic FI model with maximum speed $v_{\max }-1$. If $h<v_{\max }$ at time $t$, then the car can only move by $h$ sites in the next time-step. The FI model differs from the NS model in that the increase in speed may not be gradual, and that stochastic delay only applies to the high speed cars.

In Fig. 9, the steady state behaviour of partial densities $n_{k}(n)$ and traffic flow $q(n)$ is shown for the FI models with $v_{\max }=1$ (first row) and $v_{\max }=2$ (second row) for stochastic delay values $p=0.00,0.25$ and 0.50 . The diagrams were obtained by means of computer simulations carried out using a 860 -sites lattice with periodic boundary conditions. For density values increasing from zero to 1 with steps of $\Delta n=0.01$, the system was allowed to evolve for 1000 timesteps, and each simulation run was repeated 20 times. The results showed that, in both cases, $v_{\max }=1$ and $v_{\max }=2$, when $p=0$ the system remained under a free-flowing regime (all the particles moving with the maximum speed) until density $n$, growing from zero, reached the critical densities $n_{c}=1 / 2$ and $n_{c}=1 / 3$, respectively. The results in the top row of Fig. 9 show that FI and NS models are equivalent to each other 
when $v_{\max }=1$, independently of $p$; and they both are equivalent to WR184 model for $p=0$. For the models with $v_{\max }=2$, comparison of the top row of Fig. 8 with the second row of Fig. 9 show important differences between the respective simulations with the models FI and NS. In the limit $p=0$, the behaviour of the partial densities of the FI model as functions of the global density $n$ is quite similar, although different, to the respective behaviour of the partial densities of the NS model. However, for $p>0$ these models behave quite different from each other. For example, while in the NS model all the partial densities $n_{0}, n_{1}$ and $n_{2}$ are, in general, greater than zero for any density value $0<n<1$, in the FI model $n_{0}=0, n_{1}>0$ and $n_{2}>0$ for $0<n<1 / 2$, but $n_{0}>0, n_{1}>0$ and $n_{2}=0$ when $1 / 2<n<1$. As it is observed in Fig. 9, the FI traffic model (with $v_{\max }=2$ and $p>0$ ) switches between two different two-speed models at $n=1 / 2:\left\{n_{0}=0, n_{1}>0, n_{2}>0\right\} \leftrightarrow\left\{n_{0}>0, n_{1}>0, n_{2}=0\right\}$.

Finally, we mention the related work of Wang et al. who studied the stochastic model of Fukui and Ishibashi both analytically and numerically, providing an exact result for $p=0$, and a close approximation for the model with $p \neq 0$ (Wang et al., 1998a). Based on the FI model, they developed a model that is subtly different. They assumed that drivers do not suffer from concentration lapses at high speeds, but are instead only subjected to the random deceleration when they are driving close enough to their direct frontal leaders (Wang et al., 2001). More recently, Lee et al. incorporated anticipation with respect to a vehicle's changing space gap as its leader is driving away. This results in a higher capacity flow, as well the appearance of a synchronised traffic regime, in which vehicles have a lower speed, but are all moving (Lee et al., 2002).
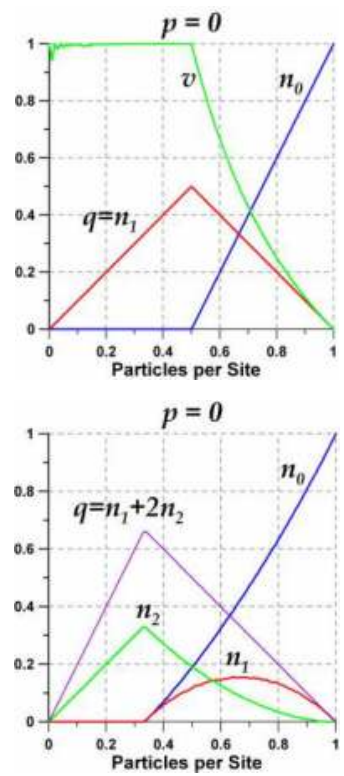

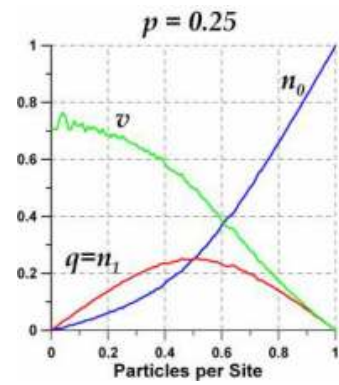

$$
p=0.25
$$

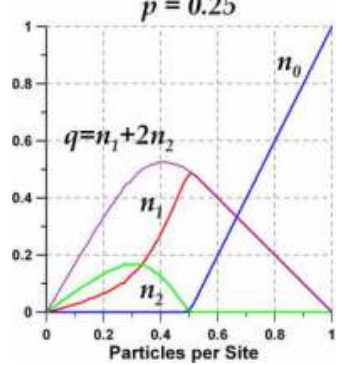

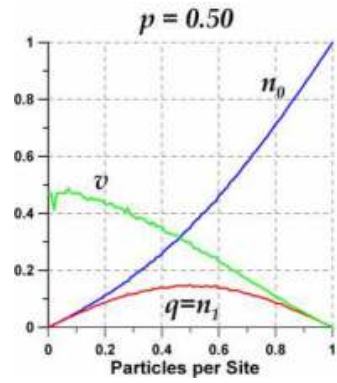

$$
p=0.50
$$

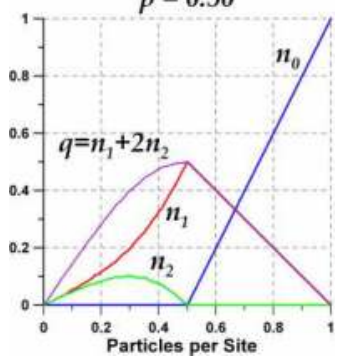

Fig. 9. The steady states of the FI models with $v_{\max }=1$ (top row) and $v_{\max }=2$ (bottom row) for stochastic delay values $p=0.00,0.25,0.50$ and 0.75 . The effect of the parameter $p$ on the traffic flow $q$, the mean speed $v$, and the partial densities $n_{k}$, as functions of $n$, is illustrated. 


\section{Equilibrium properties of the 1D traffic cellular automata}

As we have mentioned in Section 2, in the formulation of cellular automata traffic models, as it is the case of the WR184, NS and FI models, the interaction of the particles with each other is defined through some dynamical rules (deterministic and/or stochastic) which do not conserve the momentum and energy, and may drive the system far from equilibrium. The NS and FI models, in fact, have been considered as variants of the well-known asymmetric exclusion process (ASEP), the paradigm of the non-equilibrium systems (Schütz, 2001). As a consequence, notwithstanding their conceptual simplicity and easy construction, the analysis of the dynamics of a cellular automata traffic model is notoriously difficult in general. Big efforts are being made trying to apply the methods of non-equilibrium statistical physics to these systems, but only very few exact results have been obtained up to now. For the case of the NS model, the steady-state exact solution is known only if $v_{\max }=1$ (Schreckenberg et al., 1995; Evans et al., 1999). When $v_{\max }>1$, however, only approximations exist, and most of the existing results have been found through computer simulations (Schreckenberg et al., 1995; Nagel, 1996; Schadschneider \& Schreckenberg, 1993, 1997). In the case of the Fukui-Ishibashi model, H. Fuks has derived an expression of the average car flow as a function of time (Fuks, 1999). For the same model, Boccara has studied a variational principle and its existence for other deterministic cellular automata models of traffic flow (Boccara, 2001). More recently, Wang et al. studied the non-deterministic FI model with arbitrary speed limit and degree of stochastic delay deriving a general expression for the average car speed in the steady state, which was found in excellent agreement with numerical data (Wang et al., 1998b). Furthermore, in the deterministic setting, many of the results are still on the "physical" level. In particular, they were not able to prove the convergence to the average velocity described by the fundamental diagram starting from any initial configuration of a given particle density even for the finite system, not speaking about infinite ones defined on the integer lattice (Blank, 2005, 2008). Another also open problem is the existence of invariant measures with a given particle density in the random setting with jumps greater than 1 (Blank, 2005, 2008). Some excellent reviews have been published in the last decade concerning the state of the art of traffic cellular automata theory (Chowdhury et al., 2000; Helbing, 2001; Nagatani, 2002; Nagel et al., 2003; Maerivoet \& De Moor, 2005), however, up to the author's knowledge, no study was reported about the equilibrium properties of the vehicle lattice gas prior to the paper published by Salcido in 2007 (Salcido, 2007).

If we know what the equilibrium states of a system are, then we can certainly know when this system is out of equilibrium, but this may not be true in reverse sense. In the theories of thermodynamics and statistical thermodynamics, once the entropy function of the system is known, the equilibrium states of the system can be defined as all those states which maximize entropy under certain conditions. For the NS and FI models, however, detailed balance condition is not obeyed (which, otherwise, is a condition for the system can be in thermodynamical equilibrium) and so, ordinary statistical mechanics is not applicable to study them. This is what we mean when saying that the rules defining NS and FI models continuously are driving the system out of equilibrium, and one can never see relaxation towards equilibrium states. But, if we introduce constraints that prevent a system of reaching equilibrium states in practice, it does not mean, at all, that the system has no equilibrium states in theory (or better said that one cannot define equilibrium states for it). In the rest of this chapter, we will be considering a generic class of one-dimensional cellular automata models for multi-speed traffic flow with periodic boundary conditions (hereafter 
referred as GC-1DTCA). We will assume that each model in this class has all the common basic features we described in Section 2.2.1, but no particular neither explicit specification of the dynamical updating rules of the model will be done. About these rules, we just will assume that they conserve the number of particles and that prevent collisions and overtaking by assigning the speed $v$ to a particle if, and only if, it has, at least, a number $v$ of free sites ahead. Within this framework, as we will see, an entropy function can be found for the models belonging to GC-1DTCA, which allows the study the properties of the equilibrium states (which here will be understood as the maximum entropy states) of the cellular automata models for multi-speed traffic flow in a single-lane.

After description of the model system and of the variables that will describe its state, as well as the identification of the microcanonical entropy function, the maximum entropy principle will be applied to determine the equilibrium state partial densities and the thermodynamic properties of the system, such as temperature, pressure, specific heat, and isothermal compressibility. The theoretical partial densities of the allowed velocities and fundamental diagrams will be compared with computer simulation results we obtained with the NagelSchreckenberg and Fukui-Ishibashi probabilistic cellular automata traffic models. In particular, as a part of this comparison, it is shown that, although the NS and FI traffic models behave as non-equilibrium systems, they evolve rapidly towards steady states (at least under periodic boundary conditions) which we have found very close to equilibrium under the view of our theoretical framework.

\subsection{Entropy and maximum entropy states of 1D traffic cellular automata}

Our system is a traffic cellular automaton defined on a 1D-lattice with $L$ sites. It is assumed to have all the basic features cited in Section 2.2.1, but no particular or explicit specification of the velocity updating-rules is made here. However, concerning to these rules, we assumed they conserve the number of particles, and prevent collisions and overtaking by assigning the speed $v$ to a particle if, and only if, it has, at least, a number $v$ of free sites ahead. This means, in particular, that velocity anticipation is not considered here.

With this background, a particle with speed $v\left(=0,1, . . v_{\max }\right)$ can be imagined as a brick of length $v+1$ which has to be inserted in a 1D ring lattice (the brick row under question of a ring wall). This way, at any time $t$, the model system can be considered as one row of a ring wall, made of holes and $v_{\max }+1$ types of bricks (different in length) not overlapping each other. Since the dynamical rules of a particular model may change the lengths of the bricks, under certain conditions the system could reach states where the concentration of a particular type of bricks predominates over the others. The critical density is an upper bound of the density values for which only particles with speeds up to $v$ (bricks with length $v+1$ ) can be found in the system.

$$
n_{c}(v)=\frac{1}{v+1}
$$

The macroscopic state of the system will be described by the set of velocity distribution functions $N_{v}\left(v=0,1, \ldots, v_{\max }\right)$, each defined as the number of particles with some speed $v$ in the lattice. The intensive variables defined as $n_{v}=N_{v} / L$ are global partial densities of the system. Then, the global density of the number of particles, $n=N / L$, the traffic flow (or momentum per site), $q$, and the kinetic energy per site, $\varepsilon$, of the system, are defined as 


$$
\sum_{v=0}^{v_{\max }} n_{v}=n, \quad \sum_{v=0}^{v_{\max }} v n_{v}=q, \quad \sum_{v=0}^{v_{\max }} \varepsilon_{v} n_{v}=\varepsilon
$$

Here $\varepsilon_{v}$ stands for the kinetic energy of a particle with unit mass and speed $v$. It is easy to show that flow $q$ and kinetic energy $\varepsilon$ of the system cannot exceed, respectively, the maximum values $q_{\max }(n)$ and $\varepsilon_{\max }(n)$ given by

$$
\begin{gathered}
q_{\text {max }}(n)= \begin{cases}n v_{\text {max }} & 0 \leq n \leq n_{c}\left(v_{\max }\right) \\
(1-n) & n_{c}\left(v_{\text {max }}\right) \leq n \leq 1\end{cases} \\
\varepsilon_{\text {max }}(n)= \begin{cases}\frac{1}{2} n v_{\text {max }}^{2} & 0 \leq n \leq n_{c}\left(v_{\text {max }}\right) \\
\frac{1}{2}(1-n) v_{\text {max }} & n_{c}\left(v_{\text {max }}\right) \leq n \leq 1\end{cases}
\end{gathered}
$$

in the thermodynamic limit $L \rightarrow \infty$, where $n_{c}\left(v_{\max }\right)$ is the critical density for $v=v_{\max }$. Diagrams of $q_{\max }(n)$ and $\varepsilon_{\max }(n)$ are shown in Fig. 10. In the free-flow regime, $n<n_{c}\left(v_{\max }\right)$, vehicles move with speed $v_{\max }$, and the gap between vehicles is either $v_{\max }$ or larger. In consequence, the traffic flow in this regime is $q_{\max }=n v_{\max }$. If global density is larger than the critical density, i.e. $n>n_{c}\left(v_{\max }\right)$, only $(L-N) / v_{\max }$ vehicles can move with maximum speed and, in the limit $L \rightarrow \infty$, the maximum traffic flow is given $q_{\max }=1-n$. Similarly, it can be obtained the maximum value $\varepsilon_{\max }$ of kinetic energy for both $n<n_{c}\left(v_{\max }\right)$ and $n>n_{c}\left(v_{\max }\right)$. As a consequence, the possible macroscopic states of the system are defined by those partial densities $n_{v}$ which correspond to values of particle densities $n$ and kinetic energies $\varepsilon$, ranging in the intervals $0 \leq n \leq 1$ and $0 \leq \varepsilon \leq \varepsilon_{\max }(n)$, respectively.
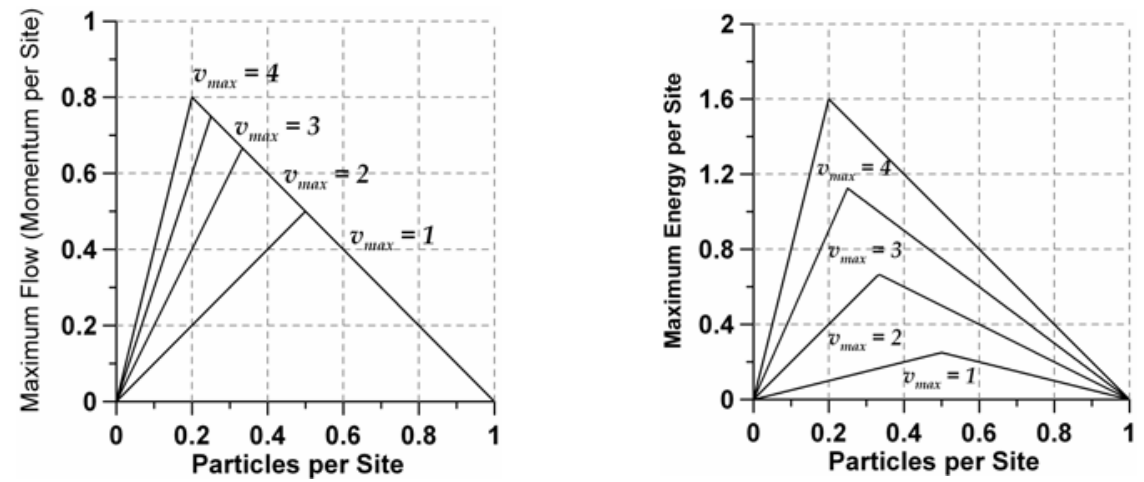

Fig. 10. Maximum flow $q_{\max }$ (left) and maximum energy $\varepsilon_{\max }$ (right) as functions of the density of particles $n$, for models with $v_{\max }=1,2,3$ and 4 . For each $n \in[0,1]$, the possible states of the system are those with energy $\varepsilon \in\left[0, \varepsilon_{\max }(n)\right]$. The transition points correspond to the critical densities $n_{c}=1 / 2,1 / 3,1 / 4$ and $1 / 5$.

Given initial and boundary conditions, the specific dynamical rules of the considered traffic cellular automata will define the macroscopic state of the system at any time $t$. Macroscopically, the state of the system will be characterized by the set of values of its partial densities $n_{v}$, or by the numbers $N_{v}=n_{v} L$, which is equivalent. Microscopically, however, there are many different arrangements in the lattice of given numbers $\left(N_{0}, N_{1}, \ldots\right.$, 
$\left.N_{v \max }\right)$ of particles moving there with speeds $\left(0,1,2, \ldots, v_{\max }\right)$, respectively, including a number $\Lambda$ of sites which must remain empty. For models belonging to the GC-1DTCA, with periodic boundary conditions, the number $\Omega\left(L, N_{v}\right)$ of all these different microscopic arrangements of moving particles is given by

$$
\Omega=\left(\frac{L}{\Lambda+N}\right)\left(\frac{(\Lambda+N) !}{\Lambda ! N_{0} ! N_{1} ! \cdots N_{v_{\max }} !}\right)
$$

and the number of empty sites in the lattice (i.e., the lattice sites available for speeding up the particles) can be expressed as

$$
\Lambda=L-\sum_{v=0}^{v_{\max }}(v+1) N_{v} \geq 0
$$

Negative values of $\Lambda$ are forbidden because of the non-anticipation restriction that we imposed to the models in the class we are considering.

We underline that $\Omega\left(L, N_{v}\right)$ is the number of all the possible configurations in which we can arrange $N_{0}$ bricks with 1-site length (representing the particles at rest), $N_{1}$ bricks with 2-sites length (representing the particles moving with speed $v=1$ ), $N_{2}$ bricks with 3-sites length (representing the particles moving with speed $v=2$ ), and so on, up to $N_{v \max }$ bricks with $\left(v_{\max }+1\right)$-sites length (particles with speed $v_{\max }$ ), by inserting them with no overlaps in a 1D ring-shaped lattice with $L$ sites in total, but allowing that a number $\Lambda$ of sites remain empty. We underline also that this result is valid for any cellular automata traffic model with all the common features we have specified above (Section 2.2.1). However, since particular dynamical rules of a model could prevent the system of reaching some microscopic states with particular configurations of particles, Eqn. (5) will provide, at least, for such cases, an upper bound to the number of them for that model.

Starting from $\Omega\left(L, N_{v}\right)$, the entropy function is defined by $S=\ln (\Omega)$. Then, with the help of Stirling's approximation, the entropy per site, $s=S / L$, in the thermodynamic limit $(L \rightarrow \infty)$, can be expressed as

$$
s=(\lambda+n) \ln (\lambda+n)-\lambda \ln \lambda-\sum_{v=0}^{v_{\max }} n_{v} \ln n_{v}
$$

where

$$
\lambda=\frac{\Lambda}{L}=1-\sum_{v=0}^{v_{\max }}(v+1) n_{v}
$$

As a pretty nice consequence of existence of entropy for the cellular automata traffic models we are considering here, we can follow microcanonical equilibrium statistical mechanics to find the equilibrium states of these models as the states that maximize the entropy for given density and energy. These constraints seem suitable because cellular automata traffic models involve rules with parameters (such as randomization $p$ in the NS-model) that control the kinetic energy of the particles, and drive the system towards macroscopic steadystates with velocity distribution densities (and kinetic energies) well defined. 
By employing the method of undetermined Lagrange multipliers, the velocity distribution functions (or partial densities) $n_{v}$ which define the equilibrium states were obtained

$$
n_{v}=\left(\frac{\lambda}{\lambda+n}\right)^{v} e^{-\alpha-\beta \varepsilon_{v}}
$$

where $\alpha$ and $\beta$ are Lagrange multipliers whose physical meaning is discussed in Section 5.

For $v_{\max }=1$, Eqn. (9) leads to the following expressions for the partial densities $n_{0}$ and $n_{1}$ as functions of $n$ and a energy related parameter $\gamma$,

$$
\begin{gathered}
n_{0}=n-n_{1} \\
n_{1}=\frac{1}{2}\left[1-\sqrt{1-4 n(1-n)\left(\frac{1}{\gamma+1}\right)}\right]
\end{gathered}
$$

Parameter $\gamma$ was defined in terms of the Lagrange multiplier $\beta$ which, as we will see later, is the conjugated variable of the global energy density (kinetic energy per site) of the system:

$$
\gamma \equiv e^{\beta / 2}
$$

Traffic flow, in this case, is given by $q=n_{1}$. Diagrams of equilibrium partial densities $n_{0}$ and $n_{1}$ are shown in Fig. 11 as functions of $n$ for those models in GC-1DTCA with $v_{\max }=1$, and values $\gamma=0,1 / 3,1$, and 3 . As can be seen, the equilibrium states we have found for $\gamma=0$ are equivalent to the steady states of the WR184 model (and also to the steady states of the NSmodel with randomization $p=0$, and of the FI-model with stochastic delay $p=0$ ). In fact, as we will see later, the equilibrium states given by the equations (10) and (11) are completely equivalent to the steady states of the NS-model with $v_{\max }=1$.
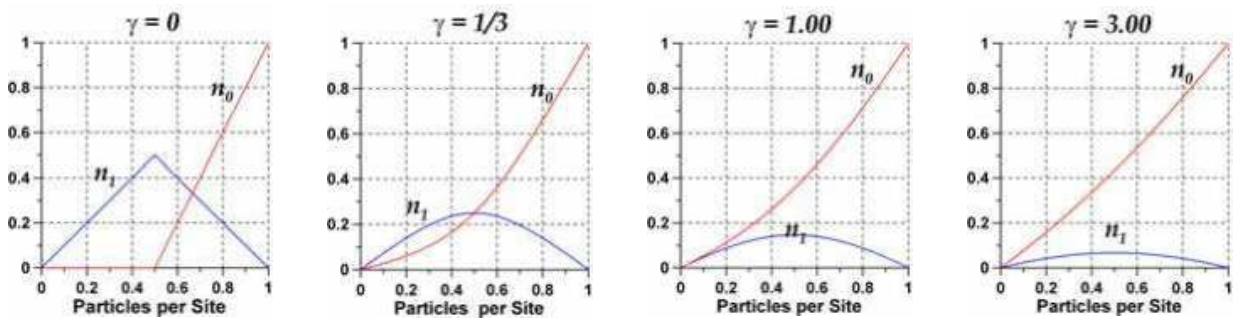

Fig. 11. Equilibrium partial densities $n_{0}$ and $n_{1}$ as functions of $n$ for models with $v_{\max }=1$. Traffic flow $q$ is the same as $n_{1}$. The effect of the parameter $\gamma$ is appreciated clearly. The equilibrium states of the models in GC-1DTCA with $v_{\max }=1$ and $\gamma=0$ are equivalent to the steady-states of models WR184, NS (with randomization $p=0$ ), and FI (with stochastic delay $p=0)$.

For $v_{\max }=2$, Eqn. (9) lead to the following equations for the velocity distribution densities:

$$
n_{0}=n-n_{1}-n_{2}
$$




$$
\begin{gathered}
n_{1}=\frac{\left(n-n_{1}-n_{2}\right)\left(1-n-n_{1}-2 n_{2}\right)}{\gamma\left(1-n_{1}-2 n_{2}\right)} \\
n_{2}=\frac{n_{1}\left(1-n-n_{1}-2 n_{2}\right)}{\gamma^{3}\left(1-n_{1}-2 n_{2}\right)}
\end{gathered}
$$

Here, given $n$ and $\gamma$, the solution of Eqns. (14) and (15) give $n_{1}$ and $n_{2}$, and then Eqn. (13) gives $n_{0}$. This system of non-linear and coupled equations can be solved with standard numerical methods (See, for example, Press et al., 1992). Here, $\gamma$ is the same as in Eqn. (12), but now traffic flow, in according to Eqn. (2), is given by $q=n_{1}+2 n_{2}$. Numerical solutions for the equilibrium partial densities $n_{0}, n_{1}$ and $n_{2}$ as functions of $n$ are shown in Fig. 12.
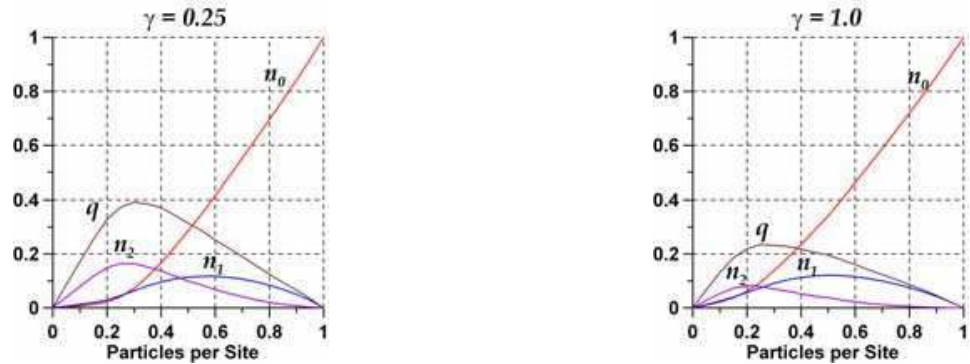

Fig. 12. Equilibrium partial densities $n_{0}, n_{1}$ and $n_{2}$ as functions of $n$ for models with $v_{\max }=2$. Traffic flow is $q=n_{1}+2 n_{2}$. The effect of the parameter $\gamma$ is appreciated clearly.

For $v_{\max }>1$, a singular behaviour of entropy at $n=n_{c}\left(v_{\max }\right)$ (see Eqn. (1)) becomes evident in the high-energy region $(\beta<0)$. This is shown in Fig. 13 for $v_{\max }=2$. For low energies $(\beta>0)$, the state of the system corresponds to arrangements of particles with the three possible speeds $\left(n_{0}, n_{1}, n_{2}>0\right)$ for all densities $0<n<1$. For high energies $(\beta<0)$, however, the number of particles with speed $v=1$ behaves as a decreasing function of energy and dies out in the high-energy limit $(\beta \rightarrow-\infty)$. Just at this point, the entropy of the system comes out

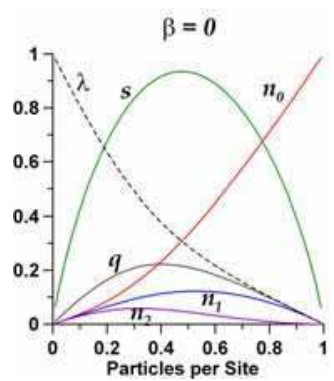

(a)

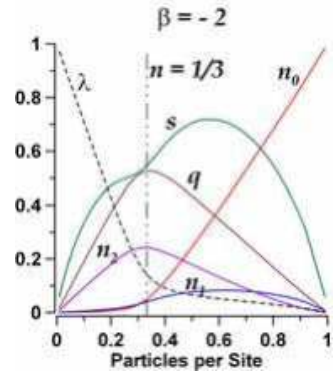

(b)

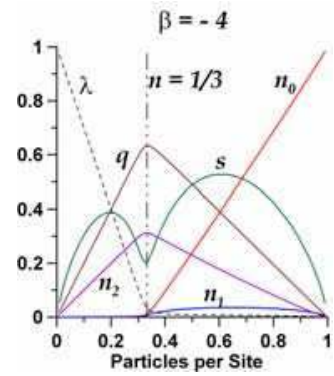

(c)

Fig. 13. Equilibrium state entropy, flow and partial densities $n_{0}, n_{1}$ and $n_{2}$ as functions of $n$ for models with $v_{\max }=2$. The behaviour of entropy, as energy increases towards its maximum $\varepsilon_{\max }(1 / 3)=2 / 3$, shows a flow-regime transition at density $n=n_{c}\left(v_{\max }\right)=1 / 3$, which becomes sharper for higher energies. 
clearly divided in two well differentiated parts: one for densities $n<1 / 3$, and the other one for densities $n>1 / 3$. The first part corresponds to free-flow states in the system, i.e., states with all particles moving with the speed $v_{\max }=2$, and a number of empty sites $\lambda>0$. The second part corresponds to congested-flow states in which the system contains only particles with speed $v_{\max }$ and particles at rest, and no empty sites $(\lambda=0)$. This behaviour of entropy suggests a flow-regime transition in the system at density $n=n_{c}\left(v_{\max }\right)=1 / 3$, which becomes sharper when energy increases towards the maximum energy $\left(\varepsilon_{\max }(1 / 3)=2 / 3\right)$.

\section{Comparison with the steady states of the NS and FI traffic models}

By comparing the simulation results shown in Figs. 4, 7 and 9, we can see that the NS and FI traffic models with $v_{\max }=1$ are identical with each other, and to the WR184 model, in the deterministic setting (i.e. when both the randomization in NS and the stochastic delay in FI are set equal to zero). As it can be observed in the first diagram of Fig. 11, the same behaviour is described by the equilibrium states for $\gamma=0$, case for which our equations (10) and (11) are reduced to

$$
\begin{gathered}
n_{0}=n-n_{1} \\
n_{1}=\frac{1}{2}[1-\sqrt{1-4 n(1-n)}]
\end{gathered}
$$

The NS and FI models, however, behave quite different each other when their respective probability parameters, randomization and stochastic delay, are larger than zero. This is, of course, what one can observe through the comparison of Fig. 7 against the diagrams shown in Fig. 9 (first row). The equilibrium states in this case $\left(v_{\max }=1, p \geq 0\right)$, result expressed by

$$
n_{0}=n-n_{1} \quad n_{1}=\frac{1}{2}[1-\sqrt{1-4 n(1-n)(1-p)}]
$$

This result is the exact solution for the NS-model with $v_{\max }=1$ (Eqn. (5.11) in Schreckenberg et al, 1995). It is obtained from Eqns. (10) and (11) once probability parameter $p$ is defined as

$$
p \equiv \frac{\gamma}{\gamma+1}=\frac{e^{\beta / 2}}{1+e^{\beta / 2}}
$$

This is consistent, of course, with the meaning of the randomization probability $p$ in the NS model because, as we will see later, the Lagrange multiplier $\beta$ is associated with the energy of the system in such a way that $\beta \rightarrow-\infty(\beta \rightarrow+\infty)$ corresponds to the high-energy (lowenergy) limit. In fact, we see that the high $(\beta \rightarrow-\infty)$ and low $(\beta \rightarrow+\infty)$ energy limits correspond to the randomization limits $p \rightarrow 0$ (no braking is allowed at all, and the particles are driven to move with the possible highest speeds) and $p \rightarrow 1$ (each particle is obligated to reduce its speed by one each timestep), respectively. In consequence, we are compelled to conclude that the equilibrium states given by the equations (10) and (11) are completely equivalent to the steady states of the NS-model with $v_{\max }=1$.

In Fig. 14, in terms of the partial densities $n_{v}$ and the traffic flow $q$, it is shown a comparison of the equilibrium states (solid lines) against computer simulations of the steady states of the NS-model (dashed lines), considering $v_{\max }=2$ and randomizations $p=0.2$ and 0.5 . Here, for each density $n$ we calculated the kinetic energy $\varepsilon$ from the partial densities $n_{v}$ of the 
simulated steady state. Then, for each couple $(n, \varepsilon)$ we solved numerically the equations (14), (15) and (16). As it can be seen, a reasonable qualitative and quantitative agreement between the equilibrium states and the steady states of NS-model is found. However, growing differences are observed as $p$ decreases below $p=0.5$, particularly for densities $n>1 / 3$.
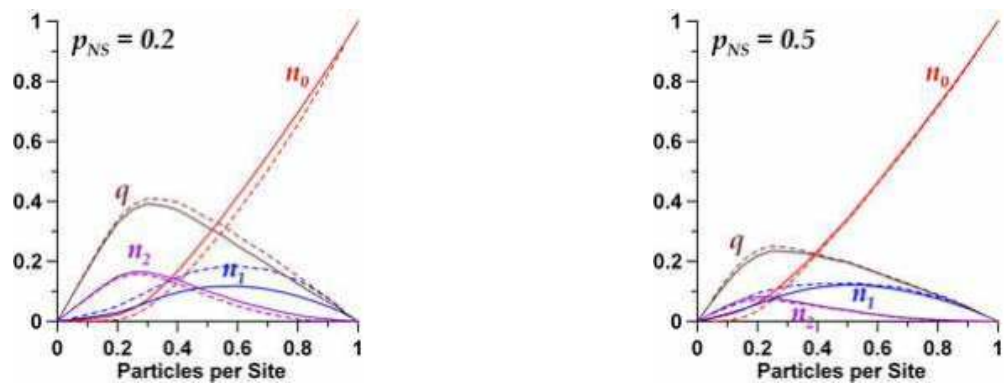

Fig. 14. Comparison between the equilibrium theory results (solid lines) and computer simulation results (dashed lines) carried out with the NS-model with $v_{\max }=2$, for randomizations $p=0.2$ (left) and 0.5 (right). Although a reasonable agreement is found, important differences are observed.

The observed differences are due to the rules implemented in the NS model for updating the speeds of the particles, which give a non-equilibrium character to the NS-model. In Fig. 15 we have shown diagrams of the deviations of the values of flow $q_{N S}$ and entropy per site $s_{N S}$ of the NS-model steady states with respect to the corresponding values $q_{E S}$ and $s_{E S}$ of the equilibrium states, for $p=0.2$ and $p=0.5$. In both cases, the NS steady-state flow deviations are positive, i.e., $q_{N S}$ is larger than $q_{E S}$ for any density $n$. For the entropy per site, however, also for any density, the values $s_{N S}$ we calculated with the partial densities of the steady states are lesser than the values $s_{E S}$ we calculated from the equilibrium ones, i.e. the
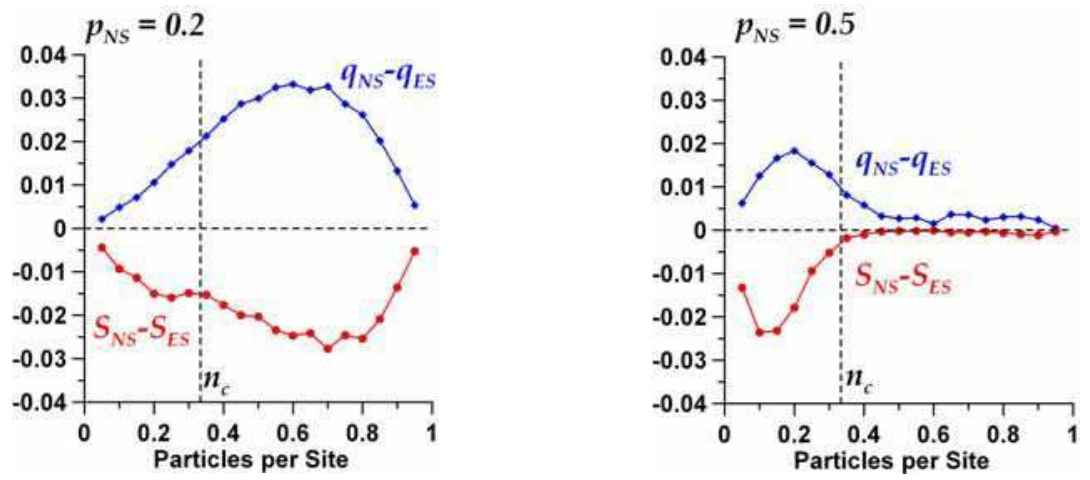

Fig. 15. Deviations of the NS steady state values of traffic flow $q$ (top) and entropy per site $s$ (bottom) with respect the corresponding equilibrium theory values, for $p=0.2$ (left) and 0.5 (right). In both cases, the NS steady-state flow is larger than that one of equilibrium. For the entropy, however, for any density $n$, the values calculated with the partial densities of the steady states are smaller than those calculated with the equilibrium ones. This result (we think) is just an expression of the non-equilibrium behaviour of the NS traffic model. 
deviations $S_{N S}-S_{E S}$ are negative. This result is exactly what we expected because of the nonequilibrium behaviour of the NS cellular automata traffic model. On another hand, as we see in these figures, for $p=0.2$, the absolute deviations $\left|s_{N S}-s_{E S}\right|$ for density values $n>n_{c}$ (congested flow regime) are larger than for $n<n_{c}$ (free-flow regime); while for $p=0.5$, on the contrary, the absolute deviations for $n<n_{c}$ are larger than for $n>n_{c}$. Furthermore, for $p=0.5$ the system behaviour in the NS-model is very close to equilibrium when $n>n_{c}$. This is due, of course, to the braking effect of the randomization parameter, which forces a better spreading of the particles among their possible speed values.

\section{Equilibrium thermodynamic properties of 1D traffic cellular automata}

In order to get some insight about the physical meaning of the Lagrange multipliers $\alpha$ and $\beta$, we note that, using Eqn. (9), the entropy can be written as

$$
s=(\alpha+\ln \lambda) n+\beta \varepsilon+\ln \left(\frac{\lambda+n}{\lambda}\right)
$$

Now, a formal comparison of this equation with the well-known Euler equation of thermodynamics for a gas of particles,

$$
s=-\frac{\mu n}{T}+\frac{\varepsilon}{T}+\frac{P}{T}
$$

where $s$ is the entropy per unit volume, $n$ is the density of the number of particles, $T$ is the temperature, $\mu$ is the chemical potential, $\varepsilon$ is the internal energy per unit volume, and $P$ is the pressure, suggests the following thermodynamics interpretation

$$
\begin{gathered}
\alpha=-\left(\frac{\mu}{T}+\ln \lambda\right) \\
\beta=\frac{1}{T} \\
\frac{P}{T}=\ln \left(\frac{\lambda+n}{\lambda}\right)
\end{gathered}
$$

Strictly speaking, Eqns. (20) and (21) just define the new parameters $T$ and $\mu$ in terms of the Lagrange multipliers $\alpha$ and $\beta$, and equation (22) defines $P$. However, the use of these properties, which we will call traffic temperature $(T)$, traffic chemical potential $(\mu)$, and traffic pressure $(P)$, could open an innovative framework for the physical analysis and interpretation of traffic flow phenomena.

Traffic temperature $(T=1 / \beta)$ may assume positive and negative values, with a discontinuity at the kinetic energy $\varepsilon_{c}$ where entropy reaches its maximum (Fig. 16), and splits the energy spectrum in a low $(T>0)$ and a high $(T<0)$ energy regions. This feature of temperature (also inherited by the traffic pressure and chemical potential) is a typical consequence of the upper bound imposed on the kinetic energy of the lattice gas particles (Salcido \& Rechtman, 1991; Bagnoli \& Rechtman, 2009), and it points out the existence of a population inversion of the particles between the low and high kinetic energies. In fact, as it is suggested by Fig. 16, 
as $n \rightarrow n_{c}\left(v_{\max }\right)=1 / 3$, entropy is zero when all the particles are at rest $\left(n_{0}=n\right)$; reaches its maximum value when the numbers of particles at rest and particles in motion are equal: $n_{0}=$ $n_{1}+n_{2}$; and becomes null again when all particles are moving with speed $v_{\max }\left(n_{2}=n\right)$.
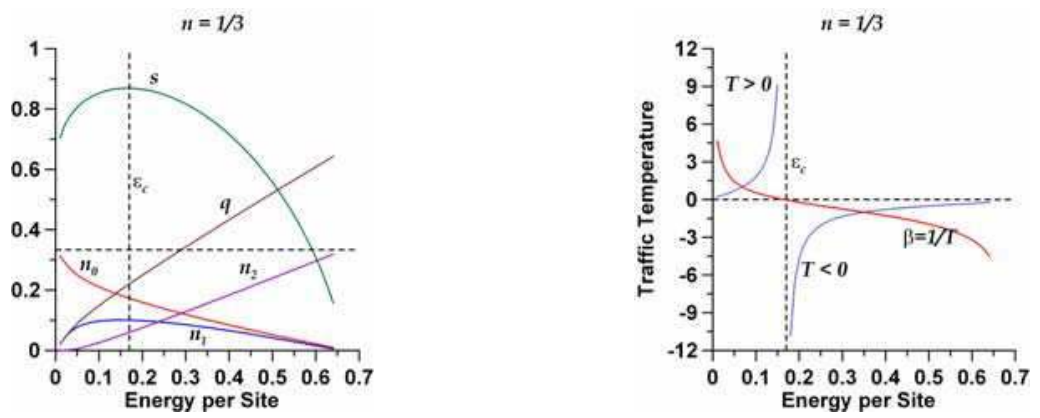

Fig. 16. (Left): The entropy per site $(s)$, flow $(q)$ and partial densities $n_{v}$ are shown as functions of energy per site $(\varepsilon)$ for the critical density $n=n_{c}\left(v_{\max }\right)=1 / 3$. Observe the typical behaviour of entropy for systems with an upper bound in energy. (Right): Temperature, defined as the slope of entropy as function of energy, has positive values for energies $\varepsilon<\varepsilon_{c}$, and negative values for $\varepsilon>\varepsilon_{c}$, being $\varepsilon_{c}$ the energy at which entropy reaches its maximum.

It is interesting the behaviour of traffic pressure in the limits $n \rightarrow 0, n \rightarrow 1$, and $n \rightarrow n_{c}\left(v_{\max }\right)$. For the first two limits, respectively, the Eqn. (22) gives

$$
\frac{P}{T} \approx n \quad \frac{P}{T} \approx \ln \left(\frac{1}{1-n}\right)
$$

The result of the first limit $(n \rightarrow 0)$ resembles the well-known equation of state of an ideal gas. In the second one $(n \rightarrow 1)$, depending on the $s$ of temperature, $P \rightarrow \pm \infty$; this result resembles the behaviour of pressure-density relation in a condensed phase. For high energies $(\beta \rightarrow-\infty)$, the same as with entropy, traffic pressure and chemical potential have a peculiar behaviour in the limit $n \rightarrow n_{c}\left(v_{\max }\right)$. This is shown in Fig. 17 for speed limit $v_{\max }=2$. In both cases, this behaviour is a result of the singularity these properties have at $\beta=0$. In the high-energy limit, the density of empty sites dies out $(\lambda \rightarrow 0)$ when density $n$ increases
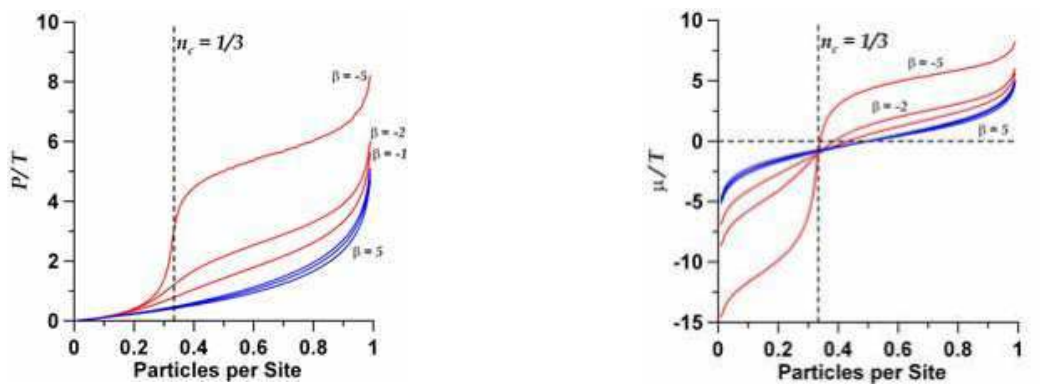

Fig. 17. Properties $P / T$ and $\mu / T$ as functions of density $n$ for several values of $\beta$. These plots suggest a critical behaviour of traffic flow near $n=n_{c}\left(v_{\max }\right)=1 / 3$ in the limit of high energy $(\beta \rightarrow-\infty)$ of the system with $v_{\max }=2$. 
towards $n_{c}\left(v_{\max }\right)$, and so $P / T$ and $\mu / T$ diverge to infinity as $\ln (1 / \lambda)$. Because $\lambda$ remains null for densities larger than $n_{c}\left(v_{\max }\right)$ (see Fig. $\left.13 \mathrm{c}\right), P / T$ and $\mu / T$ will remain undefined there.

Other thermodynamic properties, such as the specific heat $C_{v}$, isothermal compressibility $\kappa_{T}$, and isobaric expansivity $\alpha_{P}$, can be calculated easily from the velocity distribution densities. The expressions we have obtained for these thermodynamic properties are shown in the equations (24), (25) and (26). The behaviour of these properties is shown in Fig. 18.

$$
\begin{gathered}
C_{v} \equiv-\beta^{2}\left(\frac{\partial \varepsilon}{\partial \beta}\right)_{v}=\beta^{2} \sum_{v=0}^{v_{\max }} n_{v} \varepsilon_{v}^{2}-\frac{(\beta e)^{2}}{n} \\
\kappa_{T}(P, \beta) \equiv-\frac{1}{v}\left(\frac{\partial v}{\partial P}\right)_{\beta}=-\frac{n \beta e^{\beta P}}{\left(e^{\beta P}-1\right)\left(e^{\beta P}-1-n\right)} \\
\alpha_{P}(P, \beta) \equiv-\beta^{2} \frac{1}{v}\left(\frac{\partial v}{\partial \beta}\right)_{P}=\beta P \kappa_{T}(P, \beta)
\end{gathered}
$$

with

$$
v \equiv 1-\lambda=1-\frac{n}{e^{\beta P}-1}
$$

In this figure, we can observe the effect on the thermodynamic properties due to a sharp transition between the free- and congested-flow regimes. In particular, it is observed that compressibility and expansivity go to zero as $n$ is increased towards $1 / 3$. This means that for $n>1 / 3$, in the high-energy limit, no empty sites will be available in the lattice in order to set in motion the particles at rest (i.e., the system of particles cannot be expanded), and any particle will be found at rest or moving with the speed $v_{\max }$. This behaviour of particles in the high-energy limit is observed also in the Fig. 13.

\section{Conclusions and future work}

The cellular automata traffic models of Nagel-Schreckenberg (NS) and Fukui-Ishibashi (FI) include velocity updating-rules which define a dynamics that do not obey a detailed balance. These rules continuously drive the system to states out of equilibrium. This is the reason why these models and their variants cannot be studied within the framework of equilibrium statistical mechanics. Nevertheless, as we have shown here, thermodynamic entropy exists for the 1DTCA models with no velocity anticipation, which we have found through an isomorphic system where a lattice gas particle which moves with speed $v$ is modelled as a brick, $(v+1)$-sites length, that is inserted in a 1D lattice with no overlapping. This allowed us to study the equilibrium (or maximum entropy) states of these systems and their thermodynamic properties. As it could be expected, the maximum entropy states do not agree in general with the steady states of the NS model, particularly for high energies (i.e. small values of the randomization parameter $p$ ); however, in the low energies domain the equilibrium states resemble very strongly the steady states of the NS model, and the fundamental diagrams are reproduced quit well. For $v_{\max }=2$, the behaviour of entropy, as a function of the density of particles, allowed a clear identification of different flow-regimes in the 1DTCA models, displaying a sharp transition between the free- and congested-flow regimes in the high-energy limit. The presence of this transition was observed also in other 
properties of the system, such as the specific heat, the isothermal compressibility, and the isobaric expansivity, which were calculated using the velocity distribution densities of the equilibrium states. Therefore, we presume that the knowledge of thermodynamic properties within the context of modelling traffic flow by means of cellular automata is quite relevant for improving and speeding up the computer simulation of traffic flow, but also may help us to improve the physical understanding of traffic flow phenomena. So, we hope this work may contribute in advancing some modest steps in the establishment of the traffic cellular automata theory.

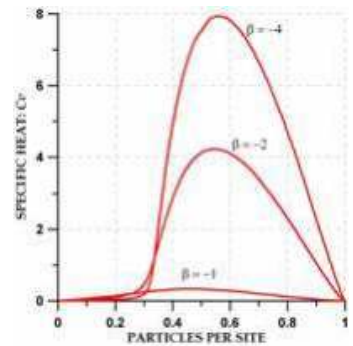

(a)

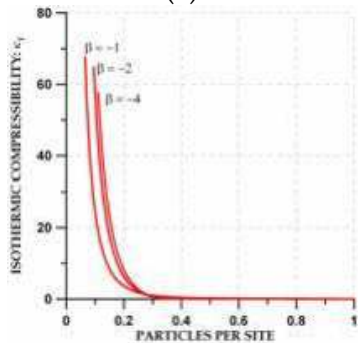

(c)

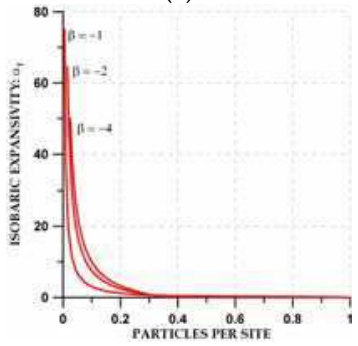

(e)

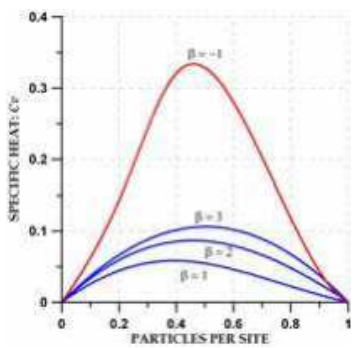

(b)

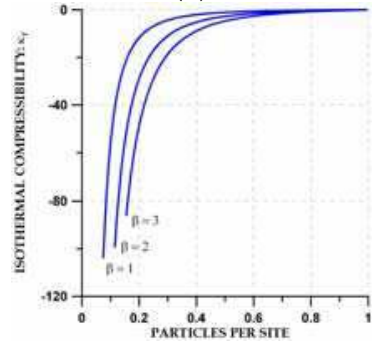

(d)

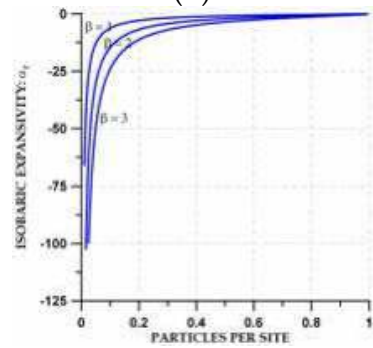

(f)

Fig. 18. Behaviour diagrams of the thermodynamic properties of the 1DTCA models with $v_{\max }=2$, for $\beta<0$ (left column) and $\beta>0$ (right column). First row: Specific heat $C_{v}$. Second row: Isothermal compressibility $\kappa_{T}$. Third row: Isobaric expansivity $\alpha_{P}$. All the properties were plotted as functions of the density of particles $n$. For $\beta<0$ these diagrams show the occurrence a flow-regime transition at density $n=n_{c}\left(v_{\max }\right)=1 / 3$. 
In the near future, we would like to be able of extending this equilibrium theory to the cellular automata models for multi-lane traffic flow and 2D traffic networks, which we hope would be setting us in the way towards the application of these models within the context of the urban air pollution problems.

\section{Acknowledgements}

We acknowledge the disinterested help and invaluable contributions we received from A. Fierros Palacios (Director, IIE-DEA, Mexico), R. Merino (TECALCO, Mexico), and A. T. Celada Murillo (IIE-DEA, Mexico) through their encouragements, comments, and enlightening discussions during the preparation of this work.

\section{References}

Bagnoli, F. (2001). Cellular Automata, In: Dynamical Modeling in Biotechnology, F. Bagnoli \& S. Ruffo (Eds.), 3-46, World Scientific, ISBN: 981-02-3604-2, Singapore.

Bagnoli, F. \& Rechtman, R. (2009). Thermodynamic entropy and chaos in a discrete hydrodynamical system, Physical Review E, Vol. 79, 041115 (April, 2009) 041115-1041115-6, ISSN: 1539-3755.

Batchelor, G. K. (1967). An Introduction to Fluid Dynamics. Cambridge University Press, ISBN13: 978-0521663960, ISBN-10: 0521663962, Cambridge.

Biham, O.; Middleton, A. A. \& Levine, D. (1992). Self-organization and a dynamical transition in traffic-flow models, Physical Review A, Vol. 46, Issue 10, (November 1992), R6124-R6127, ISSN: 1050-2947.

Boghosian, B. M. (1999). Lattice Gases and Cellular Automata, Future Generation Computer Systems, Vol. 16, Issues 2-3, (December 1999), 171-185. ISSN: 0167-739X.

Boccara, N. (2001). On the existence of a variational principle for deterministic cellular automaton models of highway traffic flow, International Journal of Modern Physics C (IJMPC), Vol. 12, Issue 2, (February 2001), 143-158, ISSN: 0129-1831.

Blank, M. (2005). Hysteresis phenomenon in deterministic traffic flows, Journal of Statistical Physics, Vol. 120, Issues 3-4, (2005), 627-658, ISSN: 0022-4715.

Blank, M. (2008). Travelling with/against the Flow. Deterministic Diffusive Driven Systems, Journal of Statistical Physics, Vol., 133, Issue 4, (2008), 773-796, ISSN: 0022-4715.

Brilon, W. \& Wu, N. (1999). Evaluation of cellular automata for traffic flow simulation on freeway and urban streets, In: Traffic andMobility: Simulation-Economics-Environment, W. Brilon, F. Huber, M. Schreckenberg, and H. Wallentowitz (Eds.), 163-180, Springer, ISBN-10: 3540662952, ISBN-13: 978-3540662952, Berlin.

Chen, S.; Lee, M.; Zhao, K. H. \& Doolen, G. D. (1989). A Lattice Gas Model with Temperature, Physica D: Nonlinear Phenomena, Vol. 37, Issues 1-3, (July 1989), 42-59, ISSN: 0167-2789.

Chopard, B. \& Droz, M. (1998). Cellular Automata Modeling of Physical Systems, Cambridge University Press, ISBN: 052146168 5, Cambridge.

Chowdhury, D.; Santen, L. \& Schadschneider, A. (2000). Statistical Physics of Vehicular Traffic and Some Related Systems. Physics Reports, Vol. 329, Issues 4-6, (May 2000) 199-329, ISSN: 0370-1573. 
Cremer, M. \& Ludwig, J. (1986). A fast simulation model for traffic flow on the basis of boolean operations, Mathematics and Computers in Simulation, Vol. 28, Issue 4 (August 1986), 297-303, ISSN: 0378-4754.

d'Humieres, D.; Lallemand, P. \& Frish, U. (1986). Lattice Gas Models for 3D Hydrodynamics, Europhysics Letters, Vol. 2, Issue 4, (August 1986), 291-297, ISSN: 0295-5075.

Domb, C. \& Lebowitz, J.L. (Eds.) (2001). Phase Transitions and Critical Phenomena, Vol. 19 (Academic Press, USA, 2001).

Doolen, G.D.; Frisch, U.; Hasslacher, B.; Orszag, S. \& Wolfram, S. (Eds). (1990). Lattice Gas Methods for Partial Differential Equations, Addison Wesley, ISBN: 020113232X, USA.

Esser, J. \& Schreckenberg, M. (1997). Microscopic Simulation of Urban Traffic based on Cellular Automata, International Journal of Modern Physics C (IJMPC), Vol. 8, Issue 5, (1997), 1025-1036, ISSN: 0129-1831.

Evans, M. R.; Rajewsky, N. \& Speer, E. R. (1999). Exact Solution of a Cellular Automaton for Traffic, Journal of Statistical Physics, Vol. 95, Issues 1-2 (April 1999), 45-96, ISSN: 0022-4715.

Frish, U.; Hasslacher, B. \& Pomeau, Y. (1986). Lattice-Gas Automata for the Navier-Stokes Equation, Physical Review Letters, Vol. 56, Issue 14, (April 1986), 1505-1508, ISSN: 1079-7114.

Fukui, M. \& Ishibashi, Y. (1993). Evolution of Traffic Jam in Traffic Flow Model, Journal of the Physical Society of Japan, Vol. 62, (1993), 3841-3844, ISSN: 0031-9015.

Fukui, M. \& Ishibashi, Y. (1996a). Traffic Flow in 1D Cellular Automaton Model Including Cars Moving with High Speed, Journal of the Physical Society of Japan, Vol. 65, (1996), 1868-1870, ISSN: 0031-9015.

Fukui, M. \& Ishibashi, Y. (1996b). Effect of reduced randomness on jam in a twodimensional traffic model, Journal of the Physical Society of Japan, Vol. 65, (1996), 1871-1873, ISSN: 0031-9015.

Fukui, M.; Oikawa, H. \& Ishibashi, Y. (1996). Flow of cars crossing with unequal velocities in a two-dimensional cellular automaton model. Journal of the Physical Society of Japan, Vol. 65, (1996), 2514-2517, ISSN: 0031-9015.

Fuks, H. (1999). Exact results for deterministic cellular automata traffic models, Physical Review E, Vol. 60, Issue 1, (July 1999), 197-202, ISSN: 1539-3755.

Gazis, D. C. (1967). Mathematical Theory of Automobile Traffic: Improved understanding and control of traffic flow has become a fast-growing area of scientific research, Science, Vol. 157, No. 3786, (July 1967) 273 - 281, ISSN: 0036-8075.

Hardy, J.; de Pazzis, O. \& Pomeau, Y. (1976). Molecular Dynamics of a Classical Lattice Gas: Transport Properties and Time Correlation Functions, Physical Review A, Vol. 13, Issue 5, (May 1976), 1949-1961, ISSN: 1050-2947.

Hardy, J.; Pomeau, Y. \& de Pazzis, O. (1973). Time Evolution of a Two-Dimensional Model System. I. Invariant States and Time Correlation Functions, Journal of Mathematical Physics, Vol. 14, Issue 12, (December 1973), 1746-1759, ISSN: 0022-2488.

Hasslacher, B. (1987). Discrete Fluids, Los Alamos Science, Vol. 15, Special Issue, (1987), 175217. Available at: www.fas.org/sgp/othergov/doe/lanl/pubs/00285743.pdf

Helbing, D. (1996). Gas-kinetic derivation of Navier-Stokes-like traffic equations, Physical Review E, Vol. 53, Issue 3, (March 1996) 2366-2381, ISSN: 1539-3755. 
Helbing, D. (1998). Structure and instability of high-density equations for traffic flow, Physical Review E, Vol. 57, Issue 5, (May 1998) 6176-6179, ISSN: 1539-3755.

Helbing, D. (2001). Traffic and related self-driven many-particle systems, Reviews of Modern Physics, Vol. 73, Issue 4, (October 2001) 1067-1141, ISSN: 0034-6861.

Helbing, D. \& Treiber, M. (1998). Gas-Kinetic-Based Traffic Model Explaining Observed Hysteretic Phase Transition, Physical Review Letters, Vol. 81, Issue 14, (October 1998) 3042-3045, ISSN: 0031-9007.

Herman, R. \& Gardels, K. (1963). Vehicular Traffic Flow, Scientific American, Vol. 209, Issue 6, (1963), 35-43.

Kadanoff, L. P. \& Swift, J. (1968). Transport Coefficients near the Critical Point: A MasterEquation Approach, Physical Review, Vol. 165, Issue 1, (January 1968), 310-322, ISSN: 0031-899X.

Krauß, S.; Nagel, K. \& Wagner, P. (1999). The mechanism of flow breakdown in traffic flow models, in: Proceedings of the International Symposium on Traffic and Transportation Theory (ISTTT99), Jerusalem, 1999.

Kühne, R. \& Michalopoulos, P. (1998). Continuum Flow Models, In: Traffic Flow Theory. A State of the Art Report, N. Gartner, C.J. Messner \& A.J. Rathi (Eds.), Transportation Research Board (TRB) Special Report 165, 2nd ed.

Lee, K.; Hui, P.; Mao, D.; Wang, B. H. \& Wu, Q. S. (2002). Fukui-Ishibashi traffic flow models with anticipation of movement of the car ahead, Journal of the Physical Society of Japan, Vol. 71, No. 7, (February 2002), 1651-1654, ISSN: 0031-9015.

Maerivoet, S. \& De Moor, B. (2005). Cellular automata models of road traffic, Physics Reports Vol. 419, Issue 1, (November 2005), 1-64, ISSN; 0370-1573.

MM5. (2003). MM5 Community Model. Visit: http://www.mmm.ucar.edu/mm5/

Nagatani, T. (1997a). Kinetic segregation in a multilane highway traffic flow, Physica A Vol. 237, Issues 1-2, (March 1997), 67-74, ISSN: 0378-4371.

Nagatani, T. (1997b). Gas Kinetics of Traffic Jam, Journal of the Physical Society of Japan, Vol. 66, (1997), 1219-1224, ISSN: 0031-9015.

Nagatani, T. (2002). The physics of traffic jams, Reports on Progress in Physics, Vol. 65, Issue 9, (September 2002), 1331-1386, ISSN: 0034-4885.

Nagel, K. (1996). Particle hopping models and traffic flow theory, Physical Review E, Vol. 53, Issue 5, (May 1996), 4655-4672, ISSN: 1539-3755.

Nagel, K. \& Barrett, C. L. (1997). Using Microsimulation Feedback For Trip Adaptation For Realistic Traffic In Dallas, International Journal of Modern Physics C, Vol. 8, Issue 3, (June 1997), 505-525, ISSN: 0129-1831.

Nagel, K. \& Nelson, P. (2005). A critical comparison of the kinematic-wave model with observational data, In: Transportation and Traffic Theory. Flow, Dynamics and Human Interaction, H.S. Mahmassani (Ed.), 145-163, Elsevier, ISBN: 0-08-044680-9, USA.

Nagel, K. \& Paczuski, M. (1995). Emergent traffic jams, Physical Review E, Vol. 51, Issue 4, (April 1995), 2909-2918, ISSN: 1539-3755.

Nagel, K. \& Schreckenberg, M. (1992). A cellular automaton model for freeway traffic. Journal de Physique I, France, Vol. 2, No. 12, (December 1992), 2221-2229.

Nagel, K.; Wagner, P. \& Woesler, R. (2003). Still Flowing: Approaches to Traffic Flow and Traffic Jam Modeling, Operations Research, Vol. 51, No. 5, (September-October 2003), 681-710, ISSN: 0030-364X. 
Nagel, K.; Wolf, D. E.; Wagner, P. \& Simon, P. (1998). Two-lane traffic rules for cellular automata: A systematic approach, Physical Review E, Vol. 58, Issue 2, (August 1998) 1425-1437, ISSN: 1539-3755.

Press, W.H; Teukolsky, S.A.; Vetterling, W.T. \& Flannery, B.P. (1992). Numerical Recipes in C: The Art of Scientific Computing, Chapter 9, Section 9.7, Cambridge University Press, ISBN: 0-521-43108-5, Cambridge.

Prigogine, I. \& Herman, R. (1971). Kinetic Theory of Vehicular Traffic. American Elsevier, ISBN: 0-444-00082-8, New York.

Rechtman, R. \& Salcido, A. (1996). Lattice Gas Self Diffusion in Random Porous Media, Fields Institute Communications, Vol. 6, (1996), 217-225, ISSN: 1069-5265.

Rechtman, R.; Salcido, A. \& Bagnoli, F. (1990). Thermomechanical Effects in a NineVelocities Two-Dimensional Lattice Gas Automaton, In: Lectures on Thermodynamics and Statistical Mechanics, M. López de Haro and C. Varea (Eds.), 182-200, World Scientific., ISBN 981-02-0243-1, Singapore.

Rechtman, R.; Salcido, A. \& Bagnoli, F. (1992). Some Near-Equilibrium Properties of a NineVelocities Lattice Gas Automaton for Two-Dimensional Hydrodynamics, In: Complex Dynamics, R. Livi, J-P. Nadal and N. Packard (Eds.), 133-139, Nova Science Publishers Inc., ISBN: 1560720182, New York.

Rickert, M. \& Nagel, K. (1997). Experiences with a Simplified Microsimulation for the Dallas/Fort-Worth Area, International Journal of Modern Physics C, Vol. 8, Issue 3, (June 1997), 483-503, ISSN: 0129-1831.

Rothery, R. W. (1998). Car Following Models. In: Traffic Flow Theory. A State of the Art Report, N. Gartner, C.J. Messner \& A.J. Rathi (Eds.), Transportation Research Board (TRB) Special Report 165, 2nd ed.

Rothman, D. \& Zaleski, S. (1997). Lattice-Gas Cellular Automata, Simple Models of Complex Hydrodynamics, Cambridge University Press, ISBN: 0-521-55-201-X, Cambridge.

Salcido, A. (1993). Lattice Gas Model for Transport and Dispersion Phenomena of Air Pollutants, In: Transactions on Ecology and the Environment, Vol. 1, P. Zannetti, C.A. Brebbia, J.E. Garcia Gardea and G. Ayala Milian (Eds.), 173-181, WIT Press, ISSN: 1743-3541, Southampton.

Salcido, A. (1994). First Evaluations of a Lattice Gas Approach to Air Pollution Modelling. In: Transactions on Ecology and the Environment, Vol. 3, J. M. Baldasano, C. A. Brebbia, H. Power and P. Zannetti (Eds.), 141-150, WIT Press, ISSN 1743-3541, Southampton.

Salcido, A. (2007). The Maximum Entropy States of 1D Cellular Automata Traffic Models, Proceedings of the 18th IASTED International Conference on Modelling and Simulation, 160-165. Montreal, Canada. 2007. ACTA Press Anaheim, CA, USA. ISBN: 978-088986-664-5.

Salcido, A. \& Celada-Murillo, A. T. (2010). A Lattice Gas Approach to the Mexico City Wind Field Estimation Problem. In: Modelling, Simulation and Optimization, G. Romero Rey and L. Martinez Munela (Eds.), 385-416, In-The, ISBN: 978-953-307-048-3, Croatia.

Salcido, A.; Celada-Murillo, A. T. \& Castro, T. (2008). Lattice Gas Simulation of Wind Fields in the Mexico City Metropolitan Area, Proceedings of the 19th IASTED International Conference on Modelling and Simulation (MS 2008), 95-100, ISBN: 9780889867413, Quebec, Canada, May 2008, Acta Press, Anaheim, Calgary, Zurich. 
Salcido, A.; Merino, R. \& Saldaña, R. (1993). Lattice Gas Model for Wind Fields over Complex Terrains. Proceedings of the International Symposium on Heat and Mass Transfer in Energy Systems and Environmental Effects, 526-531, Cancun, Mexico, August 1993, International Centre for Heat and Mass Transfer, Cancun.

Salcido, A. \& Rechtman, R. (1991). Equilibrium properties of a cellular automaton for thermofluid dynamics. In: Nonlinear Phenomena in Fluids, Solids and Other Complex Systems, P. Cordero and B. Nachtergaele (Eds), 217-229, Elsevier, ISBN: 0444887911, ISBN-13: 9780444887917, Amsterdam.

Salcido, A. \& Rechtman, R. (1993). Lattice Gas Simulations of Flows Through TwoDimensional Porous Media, Proceedings of the International Symposium on Heat and Mass Transfer in Energy Systems and Environmental Effects, 222-226, Cancun, Mexico, August 1993, International Centre for Heat and Mass Transfer, Cancun.

Sasvári, M. \& Kertész, J. (1997). Cellular automata models of single-lane traffic, Physical Review E, Vol. 56, Issue 4, (October 1997), 4104-4110, ISSN: 1539-3755.

Schadschneider, A. (1999). The Nagel-Schreckenberg model revisited, The European Physical Journal B, Vol. 10, Number 3, (August_I 1999), 573-582, ISSN: 1434-6028.

Schadschneider, A. \& Schreckenberg, M. (1993). Cellular automaton models and traffic flow, Journal of Physics A: Mathematical and General, Vol. 26, Issue 15, (August 1993), L679L684, ISSN: 0305-4470.

Schadschneider, A. \& Schreckenberg, M. (1997). Car-oriented mean-field theory for traffic flow models, Journal of Physics A: Mathematical and General, Vol. 30, Issue 4, (February 1997), L69-L75, ISSN: 0305-4470.

Schreckenberg, M.; Schadschneider, A.; Nagel, K. \& Ito, N. (1995). Discrete stochastic models for traffic flow, Physical Review E, Vol. 51, Issue 4, (April 1995), 2939-2949, ISSN: 1539-3755.

Schütt, H. (1991). Entwicklung undErprob ung eines sehr schnellen, bitorientierten Verkehrssimulationssystems für Straßennetze, Technical Report No. 6, Schriftenreihe der AG Automatisierungstechnik, T.U. Hamburg, Hamburg, 1991.

Sciarretta, A. (2006). A lattice gas model with temperature and buoyancy effects to predict the concentration of pollutant gas released by power plants and traffic sources, Mathematical and Computer Modelling of Dynamical Systems, Vol. 12, Issue 4, (August 2006), 313-327, ISSN: 1387-3954.

Sciarretta, A. \& Cipollone, R. (2001). A lattice gas model for the evaluation of transport and diffusion parameters of stack emissions in air. In: Air Pollution IX, G. Latini and C. A. Brebbia (Eds), WIT Press, ISBN: 1853128775, Southampton.

Sciarretta, A. \& Cipollone, R. (2002). On the evaluation of pollutant gas dispersion around complex sources by means of a lattice gas model. In: Air Pollution X, C. A. Brebbia and J. Martin-Duque (Eds.), 33-42, WIT Press, ISBN: 185312916X, Southampton.

Schütz, G. M. (2001). Exactly Solvable Models for Many-Body Systems Far from Equilibrium, In: Phase Tansitions and Critical Phenomena, Vol. 19, C. Domb and J.L. Lebowitz (Eds.), 1-251, Academic Press, ISBN: 0-12-220319-4, San Diego, Ca, USA.

Simon, P. M. \& Nagel, K. (1998). A Simplified Cellular Automaton Model for City Traffic, Physical Review E, Vol. 58, Issue 2, (August 1998), 1286-1295, ISSN: 1539-3755.

SMA-GDF (2008). Inventario de Emisiones de Contaminantes Criterio de la Zona Metropolitana del Valle de México, 2006. Primera Edición. Secretaría del Medio Ambiente. Gobierno del Distrito Federal. México. Document available from: 
http://www.sma.df.gob.mx/sma/links/download/archivos/ie06_criterio_pw23o ct08.pdf

Stauffer, D. (2001). Cellular Automata: Applications. In: Vector and Parallel ProcessingVECPAR 2000, J. Palma, J. Dongarra \& V. Hernández (Eds.), Lecture Notes in Computer Science, Vol. 1981/2001, (2001), 199-206, ISSN: 0302-9743. Springer Berlin/Heidelberg.

Toffoli, T. (1984). Cellular automata as an alternative to (rather than an approximation of) differential equations in modeling physics, Physica D, 10, 1, (January 1984) 117-127, ISSN: 0167-2789.

von Neumann, J. (1951). The general and logical theory of automata. In: Cerebral Mechanisms in Behaviour: The Hixon Symposium, L.A. Jeffress (Ed.), (1951), John Wiley, New York. (Reprinted in J. von Neumann, Collected Works, A. H. Taub (Ed.), Vol. 5, (1963), 288-328, Pergamon Press, New York)

von Neumann, J. (1966). Theory of Self-Reproducing Automata, Edited and completed by A.W. Burks University of Illinois Press, ISBN: 0252727339 (ISBN13: 9780252727337), Urbana, Illinois.

Vilar, L. C. Q. \& de Souza, A. M. C. (1994). Cellular automata models for general traffic conditions on a line, Physica A: Statistical Mechanics and its Applications, Vol. 211, Issue 1, (October, 1994), 84-92, ISSN: 0378-4371.

Wagner, P.; Nagel, K. \& Wolf, D. E. (1997). Realistic multi-lane traffic rules for cellular automata, Physica A: Statistical Mechanics and its Applications, Vol. 234, Issue 3-4, (January 1997), 687-698, ISSN: 0378-4371.

Wang, B. H.; Kwong, Y.R. \& Hui, P. M. (1998a). Statistical mechanical approach to FukuiIshibashi traffic flow models, Physical Review E, Vol. 57, Issue 3, (March, 1998), 2568-2573, ISSN: 1539-3755.

Wang, L.; Wang, B. H. \& Hu, B. (2001). A cellular automaton traffic flow model between the Fukui-Ishibashi and Nagel-Schreckenberg models, Physical Review E, Vol. 63, Issue 5, (April, 2001), [5 pages]: 056117, ISSN: 1539-3755.

Wang, B. H., Wang, L. \& Hui, P.M. (1997). One-Dimensional Fukui-Ishibashi Traffic Flow Model, Journal of the Physical Society of Japan, Vol. 66, No. 11, (November 1997) 36833684, ISSN: 0031-9015.

Wang, B. H.; Wang, L.; Hui, P. M. \& Hu, B. (1998b). Analytical results for the steady state of traffic flow models with stochastic delay, Physical Review E, Vol. 58, Issue 3, (September 1998), 2876-2882, ISSN: 1539-3755.

Wolfram, S. (1984). Universality and Complexity in Cellular Automata, Physica D : Nonlinear Phenomena, Vol. 10, Issues 1-2, (January, 1984), 1-35, ISSN: 0167-2789.

Wolfram, S. (1986a). Cellular Automaton Fluids I. Basic Theory, Journal of Statistical Physics, Vol. 45, Issues 3-4, (November 1986), 471-526, ISSN: 0022-4715.

Wolfram, S. (1986b). Theory and Applications of Cellular Automata. World Scientific, Singapore.

Wolfram, S. (1994). Cellular Automata and Complexity: Collected Papers. Addison-Wesley, Reading, Massachusetts.

Wolfram, S. (2002). A New Kind of Science. Wolfram Media, Inc, 2002.

Zannetti, P. (1990). Air Pollution Modelling. Theories, Computational Methods and Available Software, Computational Mechanics Publications, ISBN: 0442308051, Southampton, Boston, New York. 


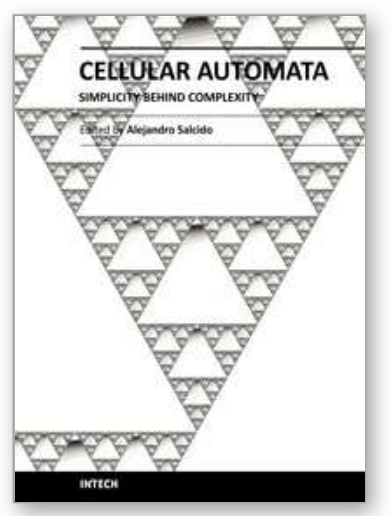

\author{
Cellular Automata - Simplicity Behind Complexity \\ Edited by Dr. Alejandro Salcido
}

ISBN 978-953-307-230-2

Hard cover, 566 pages

Publisher InTech

Published online 11, April, 2011

Published in print edition April, 2011

Cellular automata make up a class of completely discrete dynamical systems, which have became a core subject in the sciences of complexity due to their conceptual simplicity, easiness of implementation for computer simulation, and their ability to exhibit a wide variety of amazingly complex behavior. The feature of simplicity behind complexity of cellular automata has attracted the researchers' attention from a wide range of divergent fields of study of science, which extend from the exact disciplines of mathematical physics up to the social ones, and beyond. Numerous complex systems containing many discrete elements with local interactions have been and are being conveniently modelled as cellular automata. In this book, the versatility of cellular automata as models for a wide diversity of complex systems is underlined through the study of a number of outstanding problems using these innovative techniques for modelling and simulation.

\title{
How to reference
}

In order to correctly reference this scholarly work, feel free to copy and paste the following:

Alejandro Salcido (2011). Equilibrium Properties of the Cellular Automata Models for Traffic Flow in a Single Lane, Cellular Automata - Simplicity Behind Complexity, Dr. Alejandro Salcido (Ed.), ISBN: 978-953-307-230-2, InTech, Available from: http://www.intechopen.com/books/cellular-automata-simplicity-behindcomplexity/equilibrium-properties-of-the-cellular-automata-models-for-traffic-flow-in-a-single-lane

\section{INTECH}

open science | open minds

\author{
InTech Europe \\ University Campus STeP Ri \\ Slavka Krautzeka 83/A \\ 51000 Rijeka, Croatia \\ Phone: +385 (51) 770447 \\ Fax: +385 (51) 686166 \\ www.intechopen.com
}

\author{
InTech China \\ Unit 405, Office Block, Hotel Equatorial Shanghai \\ No.65, Yan An Road (West), Shanghai, 200040, China \\ 中国上海市延安西路65号上海国际贵都大饭店办公楼405单元 \\ Phone: +86-21-62489820 \\ Fax: +86-21-62489821
}


(C) 2011 The Author(s). Licensee IntechOpen. This chapter is distributed under the terms of the Creative Commons Attribution-NonCommercialShareAlike-3.0 License, which permits use, distribution and reproduction for non-commercial purposes, provided the original is properly cited and derivative works building on this content are distributed under the same license. 